\title{
S.I.: Continuous Microwave-Assisted Synthesis of Silver Nanoclusters Confined in Mesoporous SBA-15: Application in Alkyne Cyclizations.
}

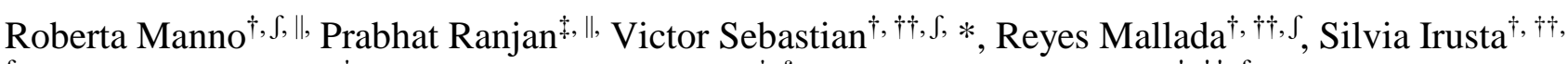
, , Upendra K. Sharma ${ }^{\ddagger}$, Erik V. Van der Eycken ${ }^{\ddagger} \S$, , and Jesús Santamaria ${ }^{\dagger}, \uparrow^{\dagger}, \rho$ 
Contents

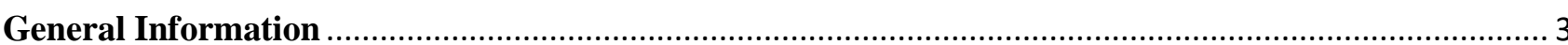

1. Optimization of heating time in batch synthesis.................................................................. 3

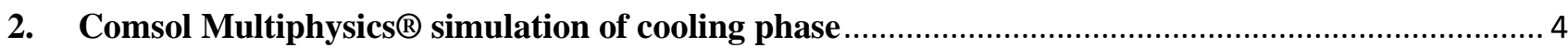

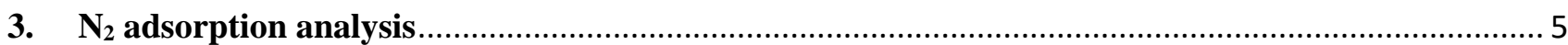

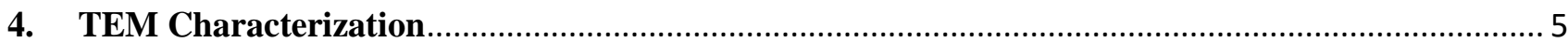

5. Reaction mechanism for hydroamination of propargylguanidines ....................................... 5

6. Leaching test for propargylguanidine cyclization ............................................................. 6

7. Catalytic reusability and stability study by reaction of 2 -(phenylethynyl)phenol (3a)..................8

8. General procedure for synthesis of starting material with corresponding characterization data..... 8

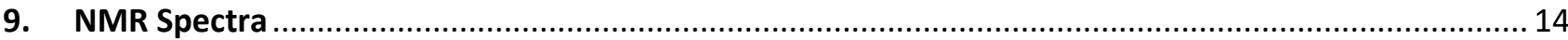




\section{General Information}

All components as well as reagents and solvents were used as received without further purification, unless stated otherwise. Reagents and solvents were bought from Sigma Aldrich and TCI and if applicable, kept under argon atmosphere. Technical solvents were bought from VWR International and Biosolve, and were used as received. Product isolation was performed using silica (60, F254, Merck ${ }^{\mathrm{TM}}$ ), and TLC analysis was performed using Silica on aluminum foils TLC plates (F254, Supelco SigmaAldrich $^{\mathrm{TM}}$ ) with visualization under ultraviolet light $(254 \mathrm{~nm}$ and $365 \mathrm{~nm})$ or appropriate TLC staining. IR spectra were recorded on Bruker Alpha FT-IR spectrometer. The IR data is presented for the maximal peaks. $1 \mathrm{H}(400 \mathrm{MHz}$ or $600 \mathrm{MHz}), 13 \mathrm{C}(100 \mathrm{MHz})$ NMR spectra were recorded on ambient temperature using a Bruker-Avance 400. $1 \mathrm{H}$ NMR spectra are reported in parts per million (ppm) downfield relative to $\mathrm{CDCl} 3(7.26 \mathrm{ppm})$ and all 13C NMR spectra are reported in ppm relative to CDCl3 (77.2 ppm). NMR spectra uses the following abbreviations to describe the multiplicity: $\mathrm{s}=$ singlet, $\mathrm{d}=$ doublet, $\mathrm{t}=$ triplet, $\mathrm{q}=\mathrm{quartet}$, $\mathrm{p}=$ pentet, $\mathrm{h}=$ hextet, hept $=$ heptet, $\mathrm{m}=$ multiplet, $\mathrm{dd}=$ double doublet, $\mathrm{td}=$ triple doublet. Known products were characterized by comparing to the corresponding $1 \mathrm{H}$ NMR and 13C NMR from literature. High resolution mass spectra were acquire on a quadrupole orthogonal acceleration time-of-flight mass spectrometer (Synapt G2 HDMS, Waters, Milford, MA). Samples were infused at $\mu \mathrm{l} / \mathrm{min}$ and spectra were obtained in positive (or: negative) ionization mode with a resolution of 15000 (FWHM) using leucine enkephalin as lock mass.

\section{Optimization of heating time in batch synthesis}

Batch synthesis was conducted in CEM Microwave Discover ${ }^{\circledR}$ Cavity with 200W for 70s, 35s and 17s. As reported in figure S1, the decrease of the irradiation time strongly influenced the size distribution of the Ag NCs, recording an optimum point at 17s with an average particle diameter of $(1.6 \pm 0.7) \mathrm{nm}$. Furthermore, comparing the results with a previous work of S. Liu et al. ${ }^{1}$, optical and morphological properties of Ag clusters are strictly dependent on the polyelectrolyte adopted. In fact, PMAA-Ag clusters presented a red transparent color. Instead PAA-Ag were dispersed in a blue transparent suspension, as reported in figure S1. 

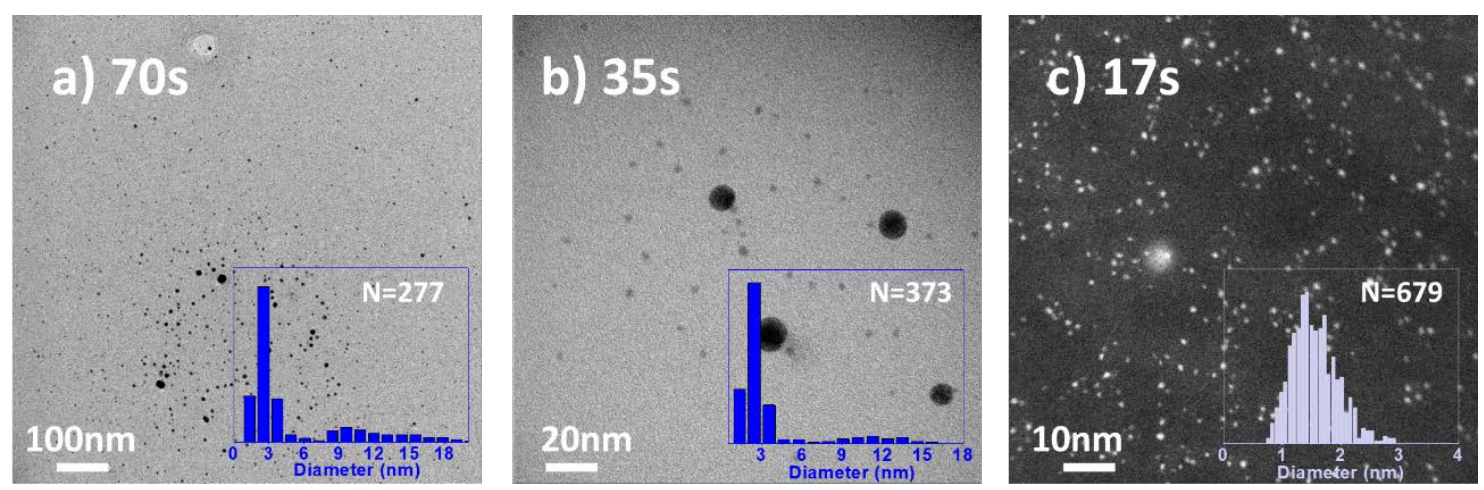

Figure S1. TEM analysis for batch synthesis of Ag NCs at respectively 70s (a), 35s (b) and 17s (c).

\section{Comsol Multiphysics ${ }^{\circledR}$ simulation of cooling phase}

Heat transfer and flow through the helically coiled reactor were evaluated from a theoretical point of view with the hypothesis of stationary state and laminar flow, a heat flux occurred between the inner wall maintained at the constant temperature of the ice bath and the flow itself.

$$
\begin{gathered}
\rho C_{p} \boldsymbol{u} \cdot \boldsymbol{\nabla} T+\boldsymbol{\nabla} \cdot \boldsymbol{q}=Q+Q_{p}+Q_{v d} \\
\boldsymbol{q}=-k \boldsymbol{\nabla} T \\
\rho(\boldsymbol{u} \cdot \boldsymbol{\nabla}) \boldsymbol{u}=\boldsymbol{\nabla} \cdot\left[-\rho \boldsymbol{l}+\mu\left(\nabla \boldsymbol{u}+(\nabla \boldsymbol{u})^{T}\right)\right]+\boldsymbol{F} \\
\rho \boldsymbol{\nabla} \cdot(\boldsymbol{u})=0
\end{gathered}
$$

The heat transfer coefficient was calculated according to equation (5) developed by Purandare et al. ${ }^{2}$ for heat transfer in helical tubes in heat exchangers:

$$
N u=0.858\left\{(D e)^{0.7202}(\operatorname{Pr})^{-1.8224}\left(\frac{a}{2 \pi R}\right)^{0.0119}\right\}=\frac{h D_{h}}{k}
$$

\begin{tabular}{|c|c|c|}
\hline Paramater & Formula & Value \\
\hline R (Coil Radius) & - & $21.185 \mathrm{~mm}$ \\
\hline r (Inner Radius) & - & $0.3875 \mathrm{~mm}$ \\
\hline a (Axial Pitch) & - & $1.5875 \mathrm{~mm}$ \\
\hline$\rho$ (density) & - & $1000 \mathrm{~kg} / \mathrm{m} 3$ \\
\hline$\mu$ (viscosity) & - & $8.9 * 10-4 \mathrm{~Pa} \cdot \mathrm{s}$ \\
\hline $\mathrm{C}_{\mathrm{P}}$ (specific heat) & - & $4185 \mathrm{~J} /(\mathrm{kg} \cdot \mathrm{K})$ \\
\hline k (thermal conductivity) & - & $0.60 \mathrm{~W} /(\mathrm{m} \cdot \mathrm{K})$ \\
\hline Dh(hydraulic diameter) & $\mathrm{D}_{\mathrm{h}}=4 \mathrm{~A} / \mathrm{P}$ & $0.3875 \mathrm{~mm}$ \\
\hline $\operatorname{Re}\left(\right.$ Reynold Number) ${ }^{245}$ & $R e=\frac{\rho v D_{h}}{\mu}$ & 274 \\
\hline
\end{tabular}

Geometrical and physical parameters adopted have been listed in Table S1. Furthermore, a detailed analysis of mesh dependence was reported in a previous work ${ }^{3}$.

Table S1. Geometrical and Physical Parameter. 


\begin{tabular}{|l|c|c|}
\hline $\operatorname{Pr}$ (Prandtl Number) $^{245}$ & $\operatorname{Pr}=\frac{c_{p} \mu}{k}$ & 0.585 \\
\hline De (Dean Number) ${ }^{245}$ & $D e=R e \sqrt{\frac{r}{R}}$ & 37 \\
\hline
\end{tabular}

3. $\mathbf{N} 2$ adsorption analysis

Table S2. $\mathrm{N}_{2}$ adsorption details for APTES/SBA-15 and Ag NCs/APTES/SBA-15

\begin{tabular}{|l|l|l|l|}
\hline Sample & SBET $\left(\mathbf{m}^{\mathbf{2}} \mathbf{g}^{-\mathbf{1}}\right)$ & $\mathbf{V}_{\mathbf{t}}\left(\mathbf{c m}^{\mathbf{3}} \mathbf{g}^{-\mathbf{1}}\right)$ & DBJH $\left._{\mathbf{n m}}\right)$ \\
\hline APTES / SBA-15 & 317 & 0.89 & 9.3 \\
\hline Ag NCs / APTES / SBA-15 & 255 & 0.59 & 9.2 \\
\hline
\end{tabular}

\section{TEM Characterization}
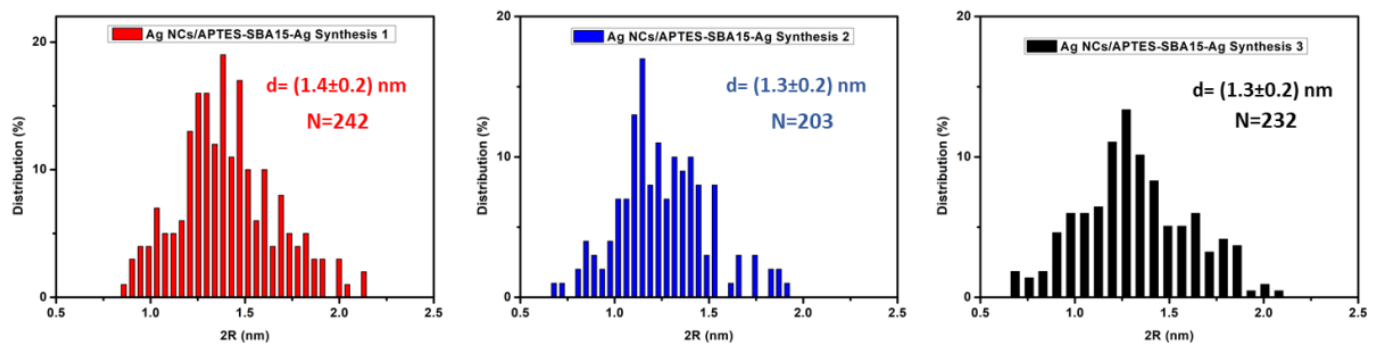

Figure S2. Reproducibility analysis of size distribution for three different synthesis of Ag NCs/APTES-SBA-15.
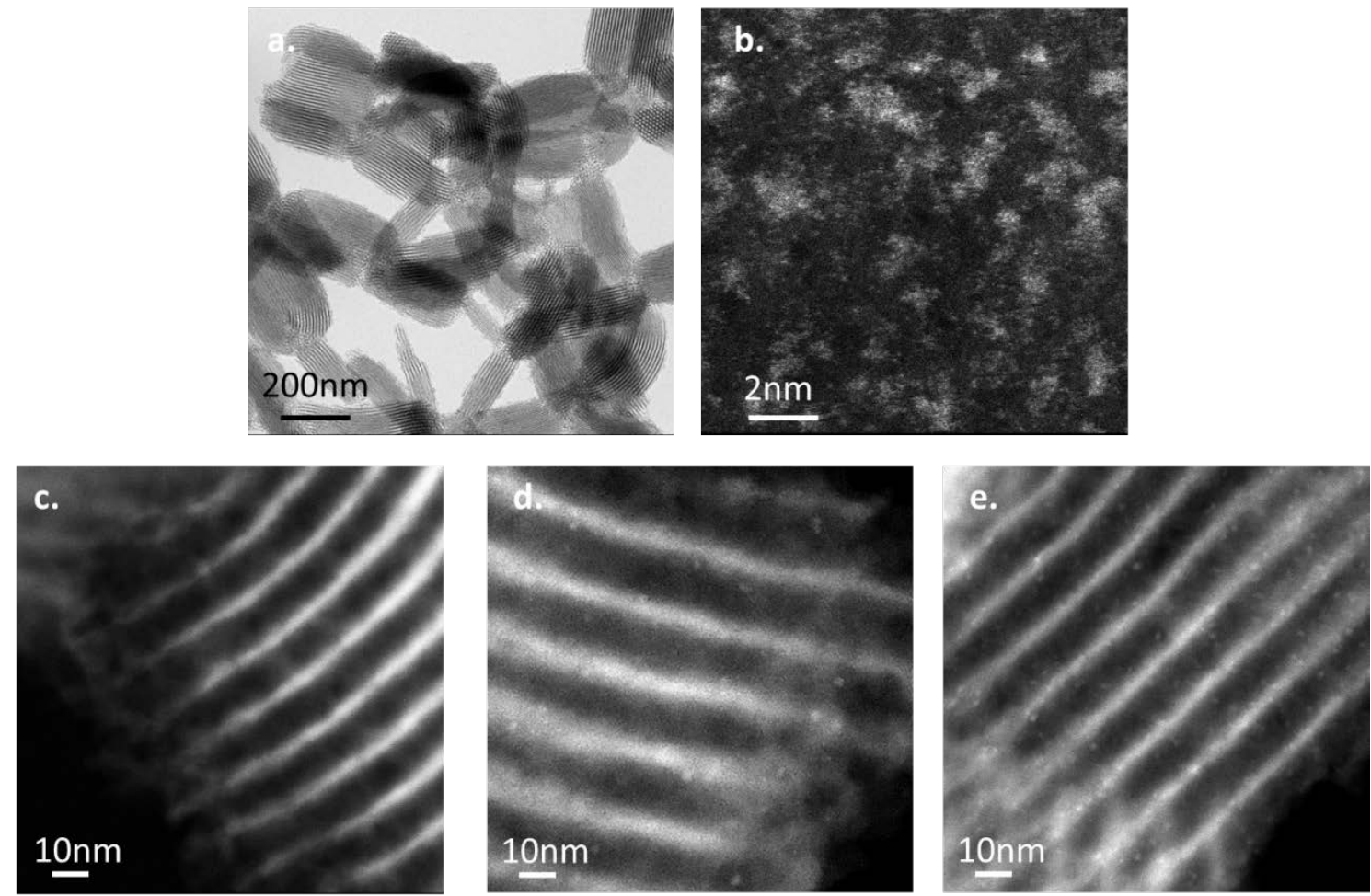

Figure S3. TEM and HRSTEM analysis for SBA15 grafted with APTES (a), unsupported Ag NCs (b), SBA15-APTES with three different loading of Ag NCs: 0.16 wt.\% (c), 0.62wt.\% (d) and 0.96wt.\% (e).

5. Reaction mechanism for hydroamination of propargylguanidines

Scheme S1: Reaction mechanism proposed for hydroamination of propargylguanidines 


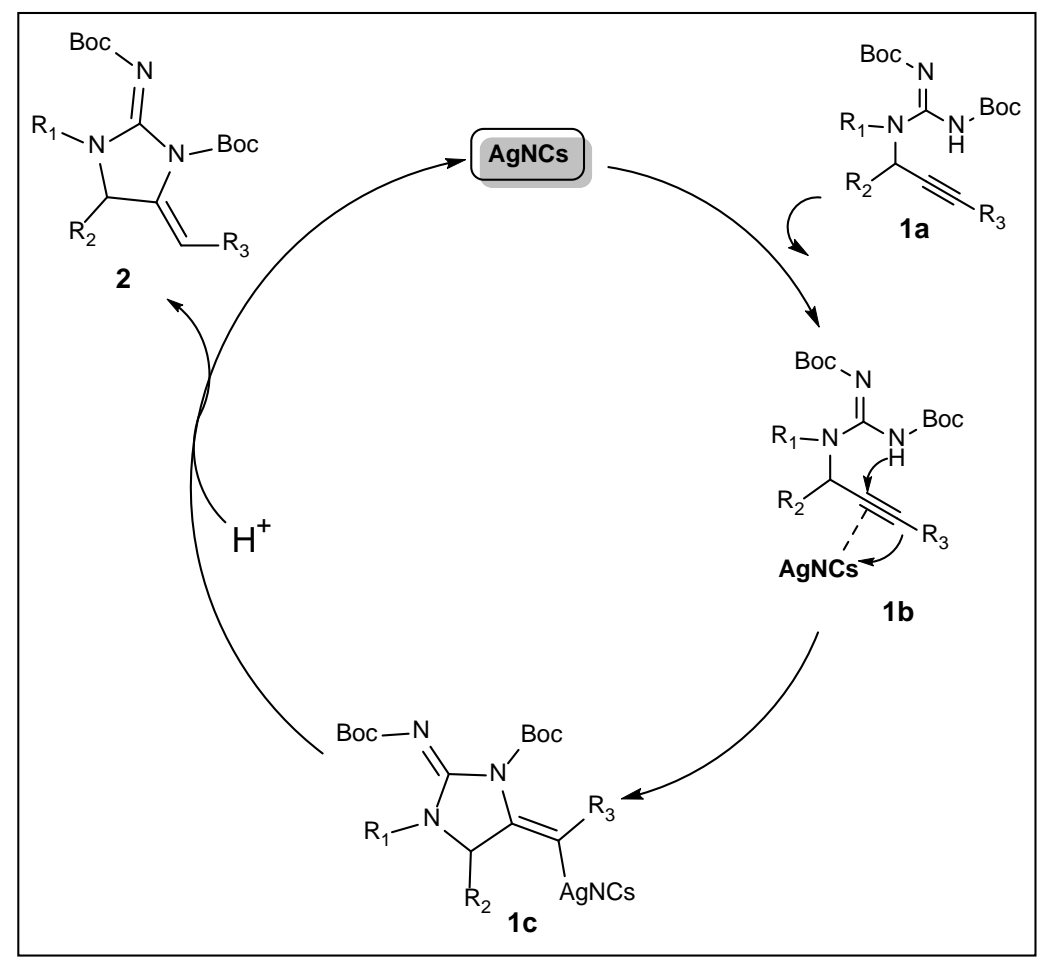

6. Leaching test for propargylguanidine cyclization
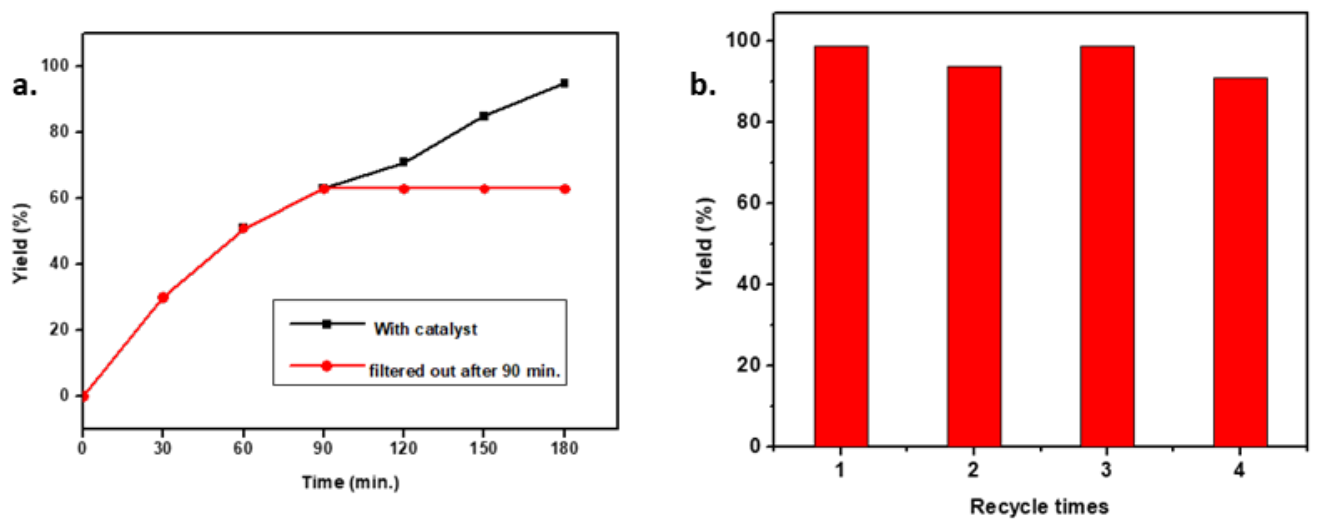

Figure S4. Leaching test analysis for Propargylguanidine cyclization. a) Hot-filtration test after 90 minutes of reaction. b) Activity tested up to 4 cycles.

Table S3. Catalyst loading determination of fresh and used catalyst by MP-AES.

\begin{tabular}{|l|l|l|}
\hline & Fresh Catalyst & Used Catalyst \\
\hline [Ag] wt\% & $(0.96+0.04) \%$ & $(0.93+0.05) \%$ \\
\hline [Ag] wt\% & $(0.62+0.03) \%$ & $(0.61+0.02) \%$ \\
\hline
\end{tabular}

Table S4. Optimization of propargylguanidine cyclization, catalyzed by Ag-NCs in continuous-flow for substrate 1f of scheme $2 .^{\mathrm{a}}$ 


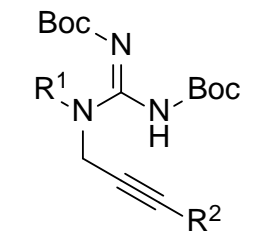

1f: $R^{1}=M e, R^{2}=P h$

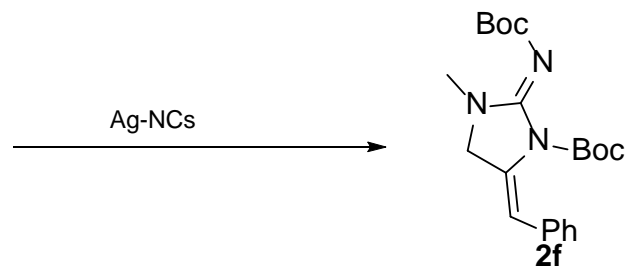

\section{Entry Ag NCs/SBA-15 Temperature Flow Rate Weight Hourly Yieldb (Conv.)

$\begin{array}{llll}(\mathrm{mg}) & \left({ }^{\circ} \mathrm{C}\right) & (\mathrm{mL} / \mathrm{min}) & \text { Space Velocity }\end{array}$

\begin{tabular}{l|llccc}
\multicolumn{1}{c}{} & \multicolumn{3}{c}{$\begin{array}{c}\text { WHSV } \\
\text { (h) }\end{array}$} \\
\hline $\mathbf{1}$ & 100 & 60 & 0.1 & 52.6 & $5(20)$ \\
$\mathbf{2}$ & 100 & 70 & 0.1 & 52.6 & $10(20)$ \\
$\mathbf{3}$ & 100 & 80 & 0.1 & 52.6 & $10(35)$ \\
$\mathbf{4}$ & 100 & 80 & 0.03 & 15.8 & $18(37)$ \\
$\mathbf{5}$ & 200 & 80 & 0.05 & 13.1 & $28(58)$ \\
$\mathbf{6}$ & 200 & 80 & 0.03 & 7.9 & $40(57)$
\end{tabular}

Experiment was performed dissolving $20 \mathrm{mg}$ of $1 \mathrm{in} 1 \mathrm{~mL}$ of toluene as per scheme 1, a Catalyst ( $0.96 \mathrm{wt} \%$ of Ag-NCs), byield was determined by NMR.
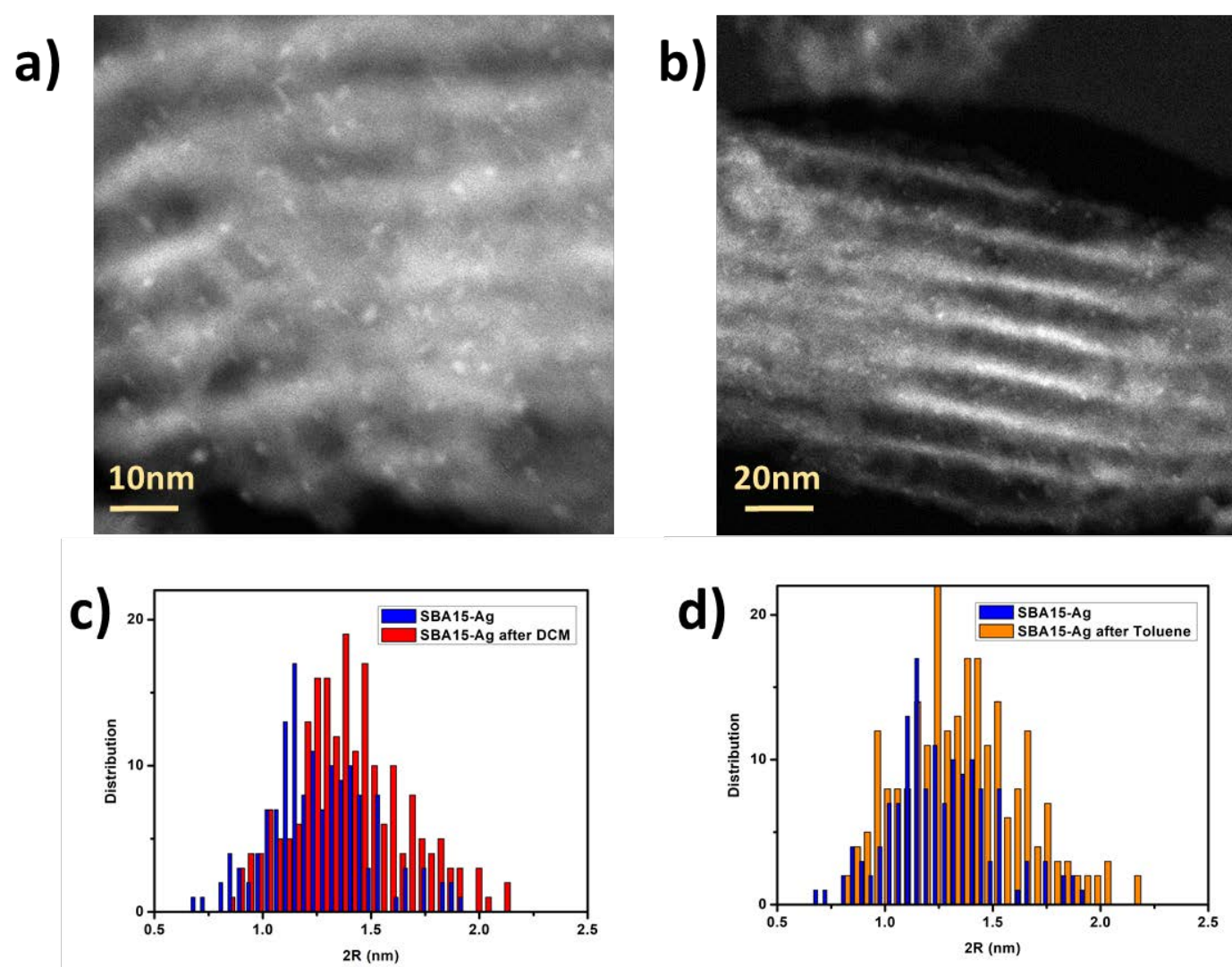

Figure S5. HRSTEM analysis for Ag NCs/SBA-15 catalyst after reaction. a) and c) after reaction at $50^{\circ} \mathrm{C}$ for $3 \mathrm{~h}$ in DCM, b) and d) after reaction at $50^{\circ} \mathrm{C}$ for $3 \mathrm{~h}$ in Toluene. 
7. Catalytic reusability and stability study by reaction of 2-(phenylethynyl)phenol (3a).
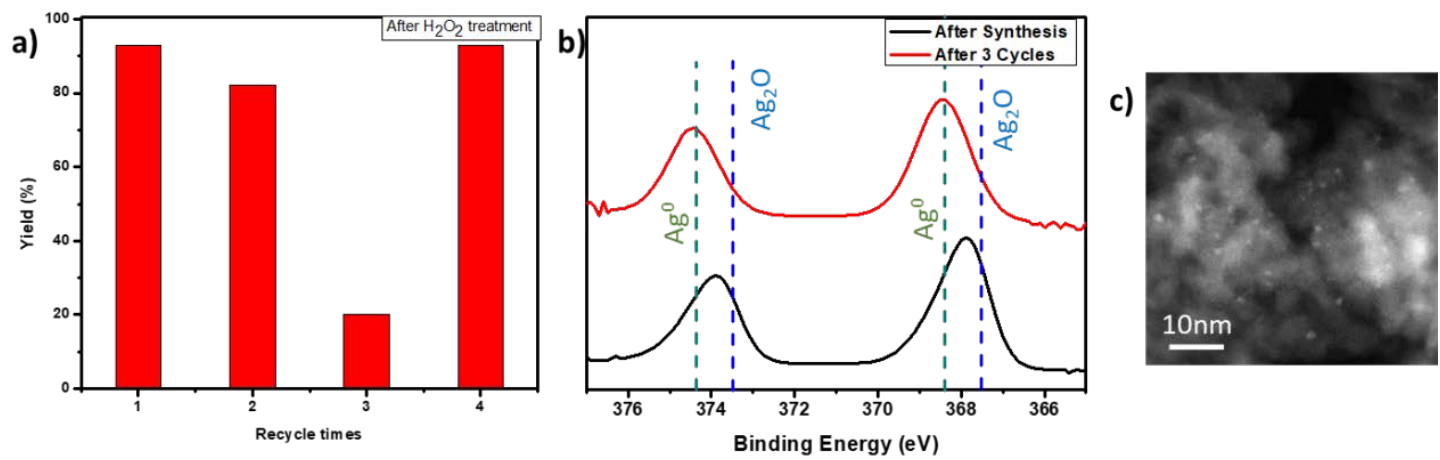

Figure S6. a) Reusability activity tested up to 4 cycle, with a $\mathrm{H}_{2} \mathrm{O}_{2}$ regeneration step. b) XPS Analysis after 3 cycles, where it is possible to notice the complete reduction of Ag. c) HR-STEM after the $\mathrm{H}_{2} \mathrm{O}_{2}$ treatment.
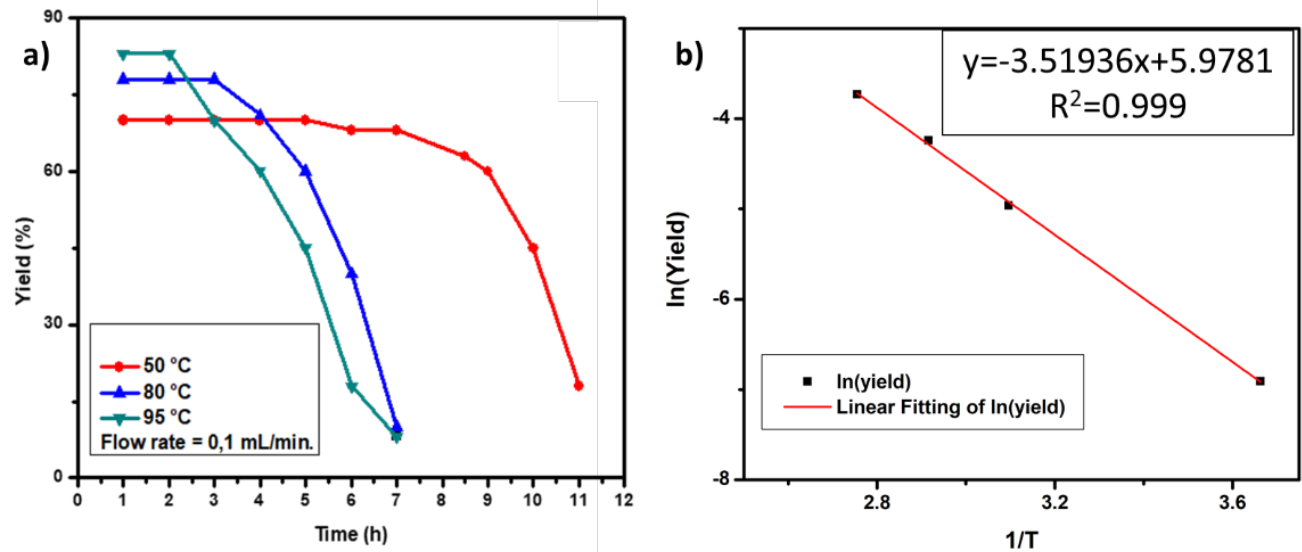

Figure S7. a) Continuous flow reaction of 2-(phenylethynyl)phenol cyclization at different heating time, $50^{\circ} \mathrm{C}, 80^{\circ} \mathrm{C}$ and $95^{\circ} \mathrm{C}$ respectively. b) Determination of the activation energy. ${ }^{6}$

\section{General procedure for synthesis of starting material with corresponding characterization data}

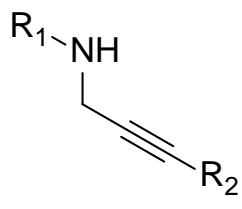

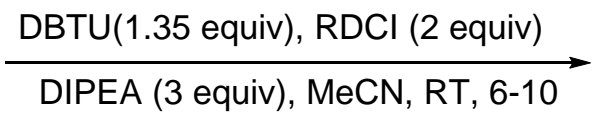<smiles>[R2]C#CCN([R1])/C(=N/[R6](=O)O[Na])NC(=O)O</smiles>

Propargylamine (0.3 mmol), 1,3-bis(tert-butoxycarbonyl)thiourea (DBTU, 115 mg,0.41 mmol, 1.35 equiv), and diisopropylethylamine (112 mg, 0.9 mmol, 3 equiv) were dissolved in anhydrous MeCN (2mL). After stirring at room temperature, it was cooled to $0{ }^{\circ} \mathrm{C}$ followed by addition of EDCI (115 mg, $0.6 \mathrm{mmol}, 2$ equiv). Then reaction was stirred at RT overnight. The reaction mixture was transferred into $250 \mathrm{~mL}$ funnel and diluted with EtOAc (50 mL), and washed with water $(3 \times 100 \mathrm{~mL})$, followed by brine and dried over anhydrous Na2SO4. The solvent was evaporated under vacuum followed by column chromatography on silica using 20\% EtOAc in heptane as eluent (Rf 0.25-0.3).

\section{(Z)-1-([1,1'-biphenyl]-4-ylmethyl)-2,3-bis(pivaloyloxy)-1-(prop-2-yn-1-yl)guanidine}




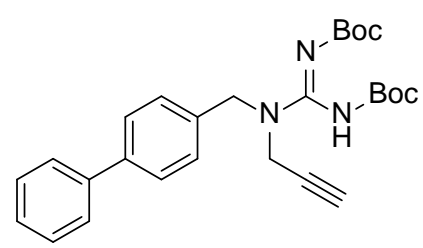

${ }^{1} \mathrm{H}$ NMR (300 MHz, Chloroform-d) $\delta 10.04$ (s, 1H), 7.58 (dd, $\left.J=7.7,3.8 \mathrm{~Hz}, 4 \mathrm{H}\right), 7.41$ (dt, $J=20.8$, $7.8 \mathrm{~Hz}, 5 \mathrm{H}), 4.84$ (s, 2H), 4.18 (d, $J=2.5 \mathrm{~Hz}, 2 \mathrm{H}), 2.35-2.29(\mathrm{~m}, 1 \mathrm{H}), 1.51(\mathrm{~s}, 18 \mathrm{H}) .{ }^{13} \mathrm{C}$ NMR $(101$ $\left.\mathrm{MHz}, \mathrm{CDCl}_{3}\right) \delta 167.59,166.59,136.94,135.70,134.35,133.54,130.08,129.09,128.98,128.58$, 128.25, 127.41, 127.35, 126.79, 125.86, 125.03, 67.98, 67.92, 66.91, 66.86, 63.82, 51.09, 42.69, 42.61, 32.39, 32.33, 18.78, 18.68, 13.70. HRMS (ESI) calculated for $\mathrm{C}_{27} \mathrm{H}_{33} \mathrm{~N}_{3} \mathrm{O}_{4}[\mathrm{M}+\mathrm{H}]^{+}:$463.24708; found: 464.2530

\section{(Z)-1-(4-methoxybenzyl)-2,3-bis(pivaloyloxy)-1-(prop-2-yn-1-yl)guanidine:}

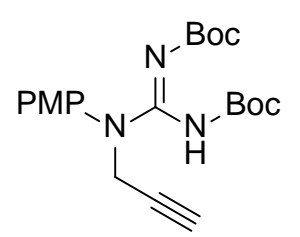

${ }^{1} \mathrm{H}$ NMR (400 MHz, Chloroform-d) $\delta 10.00$ (s, 1H), 7.24 (d, $\left.J=8.3 \mathrm{~Hz}, 2 \mathrm{H}\right), 6.86$ (d, $\left.J=8.4 \mathrm{~Hz}, 2 \mathrm{H}\right)$, 4.72 (s, 2H), 4.11 (s, 2H), 3.80 (s, 3H), 2.30 (d, $J=2.5 \mathrm{~Hz}, 1 \mathrm{H}), 1.53-1.45$ (m, 18H). ${ }^{13} \mathrm{C}$ NMR (101 $\left.\mathrm{MHz}, \mathrm{CDCl}_{3}\right) \delta 162.54,159.37,155.46,152.67,150.73,149.83,129.90,127.55,114.15,77.92,73.50$, 55.36, 50.95, 37.07, 28.24, 28.12. HRMS (ESI) calculated for $\mathrm{C}_{22} \mathrm{H}_{31} \mathrm{~N}_{3} \mathrm{O}_{5}[\mathrm{M}+\mathrm{H}]^{+}$: 417.22635; found: 418.2330.

\section{(Z)-1-benzyl-2,3-bis(pivaloyloxy)-1-(prop-2-yn-1-yl)guanidine:}<smiles>C#CCN(Cc1ccccc1)/C(=N/C(C)(C)C)NC(=O)OC(C)(C)C</smiles>

${ }^{1} \mathrm{H}$ NMR (400 MHz, Chloroform-d) $\delta 10.01(\mathrm{~s}, 1 \mathrm{H}), 7.40$ - $7.22(\mathrm{~m}, 5 \mathrm{H}), 4.82(\mathrm{~d}, J=21.6 \mathrm{~Hz}, 2 \mathrm{H})$, $4.14(\mathrm{t}, J=2.4 \mathrm{~Hz}, 2 \mathrm{H}), 2.30(\mathrm{~d}, J=2.5 \mathrm{~Hz}, 1 \mathrm{H}), 1.50(\mathrm{~s}, 18 \mathrm{H}) .{ }^{13} \mathrm{C}$ NMR $\left(101 \mathrm{MHz}, \mathrm{CDCl}_{3}\right) \delta 170.21$, 162.45, 138.07, 135.15, 129.43, 129.03, 128.91, 128.72, 128.35, 128.23, 128.07, 106.00, 80.04, 54.02, 
53.53, 50.66, 28.44, 28.31, 28.09. HRMS (ESI) calculated for $\mathrm{C}_{21} \mathrm{H}_{29} \mathrm{~N}_{3} \mathrm{O}_{4}[\mathrm{M}+\mathrm{H}]^{+}$: 387.21579 ; found: 388.2219.

(Z)-1-(cyclohexylmethyl)-2,3-bis(pivaloyloxy)-1-(prop-2-yn-1-yl)guanidine:<smiles>C#CCN(CC1CCCCC1)/C(=N/C(C)(C)C)NC(=O)OC(C)(C)C</smiles>

${ }^{1} \mathrm{H}$ NMR (300 MHz, Chloroform-d) $\delta 9.83$ (s, 1H), $4.33-4.25$ (m, 2H), 3.40 (d, J = 7.0 Hz, 2H), 2.28 (d, $J=2.5 \mathrm{~Hz}, 1 \mathrm{H}), 1.76-1.60(\mathrm{~m}, 8 \mathrm{H}), 1.48$ (s, 18H), 1.20 (s, 2H), $1.00-0.82(\mathrm{~m}, 1 \mathrm{H}) .{ }^{13} \mathrm{C}$ NMR $\left(101 \mathrm{MHz}, \mathrm{CDCl}_{3}\right) \delta 162.55,159.45,155.64,153.27,150.66,149.53,137.22,91.63,84.26,78.85$, 77.96, 73.38, 50.85, 50.04, 35.81, 35.70, 30.70, 30.64, 28.51, 28.39, 28.34, 28.29, 28.19, 26.50, 26.45, 25.88. HRMS (ESI) calculated for $\mathrm{C}_{21} \mathrm{H}_{35} \mathrm{~N}_{3} \mathrm{O} 4$ [M+H] $]^{+}$: 393.26273; found: 394.2691.<smiles>CN(CC#Cc1ccccc1)C(=NC(=O)OCc1ccccc1)NC(=O)OCc1ccccc1</smiles>

$1 f$

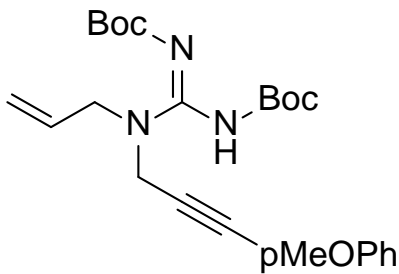

$1 \mathrm{~g}$

Analytical data: Regioselective Rhodium(II)-Catalyzed Hydroaminations of Propargylguanidines: Gainer, M. J.; Bennett, N. R.; Takahashi, Y.; Looper, R. E. Angew. Chemie - Int. Ed. 2011, 50 (3), $684-687$.

tert-butyl carboxylate:<smiles>C=C1CN(C)C(=NC(=O)OC(C)(C)C)C1N(C)C(=O)OC(C)(C)C</smiles>

${ }^{1} \mathrm{H}$ NMR (400 MHz, Chloroform-d) $\delta 5.19$ (q, $\left.J=2.2 \mathrm{~Hz}, 1 \mathrm{H}\right), 4.46(\mathrm{q}, J=2.0 \mathrm{~Hz}, 1 \mathrm{H}), 4.08(\mathrm{t}, J=$ $2.4 \mathrm{~Hz}, 2 \mathrm{H}), 2.94$ (s, 3H), 1.56 (s, 8H), 1.50 (s, 9H).

Analytical data: Ermolat'ev D. S.; Bariwal, J. B.; Steenackers, H. P. L.; De Keersmaecker, S. C. J.; 
Van Der Eycken, E. V. Angew. Chemie - Int. Ed. 2010, 49 (49), 9465-9468.

tert-butyl

(E)-3-([1,1'-biphenyl]-4-ylmethyl)-2-((tert-butoxycarbonyl)imino)-5methyleneimidazolidine-1-carboxylate:<smiles>C=C1CN(Cc2ccc(-c3ccccc3)cc2)C(=NC(=O)OC(C)(C)C)N1C(=O)OCc1ccccc1</smiles>

${ }^{1} \mathrm{H}$ NMR (400 MHz, Chloroform-d) $\delta 7.56(\mathrm{t}, J=7.4 \mathrm{~Hz}, 5 \mathrm{H}), 7.44$ (t, $\left.J=7.6 \mathrm{~Hz}, 2 \mathrm{H}\right), 7.34$ (dd, $J=$ 11.0, 7.6 Hz, 3H), 5.17 (q, $J=2.3 \mathrm{~Hz}, 1 \mathrm{H}), 4.63$ (s, 2H), 4.40 (q, $J=2.1 \mathrm{~Hz}, 1 \mathrm{H}), 3.93$ (d, $J=2.3 \mathrm{~Hz}$, 2H), 1.56 (d, $J=16.8 \mathrm{~Hz}, 18 \mathrm{H}) .{ }^{13} \mathrm{C}$ NMR (101 MHz, $\left.\mathrm{CDCl}_{3}\right) \delta 159.46,152.99,149.53,141.00$, 140.70, 137.03, 134.27, 128.92, 127.62, 127.54, 127.19, 91.88, 84.55, 79.21, 48.65, 48.28, 28.52, 28.24, 28.21. HRMS (ESI) calculated for $\mathrm{C}_{27} \mathrm{H}_{33} \mathrm{~N}_{3} \mathrm{O}_{4}[\mathrm{M}+\mathrm{H}]^{+}$: 463.24708; found: 464.2538

tert-butyl(E)-2-((tert-butoxycarbonyl)imino)-3-(4-methoxybenzyl)-5-methyleneimidazolidine1-carboxylate:<smiles>C=C1CN(NC(=O)c2ccccc2)C(=NC(=O)OC(C)(C)C)N1C(=O)OC(C)(C)C</smiles>

${ }^{1} \mathrm{H}$ NMR (300 MHz, Chloroform-d) $\delta 7.18$ (d, $\left.J=8.6 \mathrm{~Hz}, 2 \mathrm{H}\right), 6.89-6.81$ (m, 2H), 5.15 (q, $J=2.4 \mathrm{~Hz}, 1 \mathrm{H}$ ), 4.52 (s, 2H), 4.37 (q, $J=2.2 \mathrm{~Hz}, 1 \mathrm{H}), 3.86$ (t, $J=2.5 \mathrm{~Hz}, 2 \mathrm{H}), 3.79$ (d, $J=0.7 \mathrm{~Hz}, 3 \mathrm{H}), 1.57$ (s, 9H), 1.53 (s, 9H). ${ }^{13} \mathrm{C}$ NMR (75 MHz, $\left.\mathrm{CDCl}_{3}\right) \delta 159.74,159.34,152.71,149.57,137.41,129.90,127.51,114.50,91.42,84.44,78.99,55.45,48.62,48.21,28.64$, 28.29. HRMS (ESI) calculated for $\mathrm{C}_{22} \mathrm{H}_{31} \mathrm{~N}_{3} \mathrm{O}_{5}[\mathrm{M}+\mathrm{H}]^{+}$: 417.22635; found: 418.2330.

tert-butyl(E)-3-benzyl-2-((tert-butoxycarbonyl)imino)-5-methyleneimidazolidine-1carboxylate:<smiles>C=C1CN(Br)C(=NC(=O)OC(C)(C)C)C1=NC(=O)OCc1ccccc1</smiles>

${ }^{1} \mathrm{H}$ NMR (300 MHz, Chloroform-d) $\delta 7.37-7.28$ (m, 4H), 7.24 (m, 1H), $5.16(\mathrm{q}, J=2.5 \mathrm{~Hz}, 1 \mathrm{H})$, 4.58 (s, 2H), 4.38 (q, $J=2.1 \mathrm{~Hz}, 1 \mathrm{H}), 3.88$ (t, $J=2.4 \mathrm{~Hz}, 1.5 \mathrm{H}), 3.85$ (t, $J=2.4 \mathrm{~Hz}, 0.5 \mathrm{H}), 1.57$ (s, 9H), 1.52 (s, 9H). ${ }^{13} \mathrm{C}$ NMR (101 MHz, $\left.\mathrm{CDCl}_{3}\right) \delta 170.17,162.41,152.59,149.77,138.05,135.13$, 
128.88, 128.19, 128.04, 105.96, 81.92, 79.98, 53.99, 50.63, 30.95, 28.28, 28.06, 28.04. HRMS (ESI) calculated for $\mathrm{C}_{21} \mathrm{H}_{29} \mathrm{~N}_{3} \mathrm{O}_{4}[\mathrm{M}+\mathrm{H}]^{+}$: 387.21579; found: 388.2220.

tert-butyl(Z)-2-((tert-butoxycarbonyl)imino)-3-(cyclohexylmethyl)-5-methyleneimidazolidine1-carboxylate:

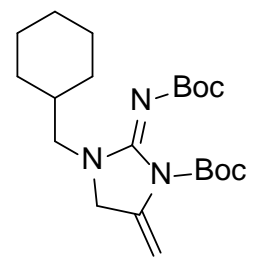

${ }^{1} \mathrm{H}$ NMR (300 MHz, Chloroform-d) $\delta 5.16(\mathrm{q}, J=2.4 \mathrm{~Hz}, 1 \mathrm{H}), 4.43(\mathrm{q}, J=2.1 \mathrm{~Hz}, 1 \mathrm{H}), 4.04(\mathrm{t}, J=$ $2.4 \mathrm{~Hz}, 2 \mathrm{H}), 3.20$ (d, $J=7.1 \mathrm{~Hz}, 2 \mathrm{H}), 1.64$ (dtd, $J=17.9,6.5,5.9,3.4 \mathrm{~Hz}, 4 \mathrm{H}), 1.54$ (s, 9H), 1.48 (s, 9H), $1.34-1.22$ (m, 4H), $1.18-1.10$ (m, 2H), 0.96 (s, 1H). ${ }^{13} \mathrm{C}$ NMR (75 MHz, Chloroform-d) $\delta$ 159.25 , 153.01 , 149.58 , 137.57 , 91.24 , 84.26 , 78.71 , 51.18 , 50.36 , 36.09 , 32.01 , 30.94 , 29.08 , $28.65,28.29$, $26.54,25.93,22.76,14.06$. HRMS (ESI) calculated for $\mathrm{C}_{21} \mathrm{H}_{35} \mathrm{~N}_{3} \mathrm{O} 4[\mathrm{M}+\mathrm{H}]^{+}$: 393.26273; found: 394.2686.

tert-butyl(E)-5-((Z)-benzylidene)-2-((tert-butoxycarbonyl)imino)-3-methylimidazolidine-1carboxylate:<smiles>CN1C/C(=C/c2ccccc2)N(C(=O)OC(C)(C)C)/C1=N/C(=O)OCc1ccccc1</smiles>

${ }^{1} \mathrm{H}$ NMR (300 MHz, Chloroform-d) $\delta 7.40$ (d, $\left.J=7.3 \mathrm{~Hz}, 2 \mathrm{H}\right), 7.31$ (t, $\left.J=7.6 \mathrm{~Hz}, 2 \mathrm{H}\right), 7.20(\mathrm{t}, J=$ $7.3 \mathrm{~Hz}, 1 \mathrm{H}), 5.99$ (d, $J=2.0 \mathrm{~Hz}, 1 \mathrm{H}), 4.05$ (d, $J=1.9 \mathrm{~Hz}, 2 \mathrm{H}), 3.02$ (s, 3H), 1.56 (s, 9H), 1.01 (s, 9H). Analytical data: Regioselective Rhodium(II)-Catalyzed Hydroaminations of Propargylguanidines: Gainer, M. J.; Bennett, N. R.; Takahashi, Y.; Looper, R. E. Angew. Chemie - Int. Ed. 2011, 50 (3), 684-687.

tert-butyl (E)-3-allyl-5-((Z)-benzylidene)-2-((tert-butoxycarbonyl)imino)imidazolidine-1carboxylate: 
<smiles>C=CCN1C/C(=C/COC(C)(C)C)C(=O)OC(C)(C)C(=O)Oc2ccccc21</smiles>

Analytical data: Regioselective Rhodium(II)-Catalyzed Hydroaminations of Propargylguanidines:

Gainer, M. J.; Bennett, N. R.; Takahashi, Y.; Looper, R. E. Angew. Chemie - Int. Ed. 2011, 50 (3), 684-687.

\section{2-(phenylethynyl)phenol}<smiles>Oc1ccccc1C#Cc1ccccc1</smiles>

\section{2-phenylbenzofuran}<smiles>c1ccc(-c2cc3ccccc3o2)cc1</smiles>

For detail analytical data: Zhao, J.; Nguyen, S. C.; Ye, R.; Ye, B.; Weller, H.; Somorjai, G. A.; Alivisatos, A. P.; Dean Toste, F. ACS Cent. Sci. 2017, 3 (5), 482-488.

N-benzyl-N-(2-(tert-butylamino)-1-(4-hydroxy-3-methoxyphenyl)-2-oxoethyl)propiolamide and 3-benzyl-8-methoxy-1-methylene-3,3a,5a,6-tetrahydro-1H-pyrrolo[2,3-c]indole-2,4,7(5H)trione respectively.
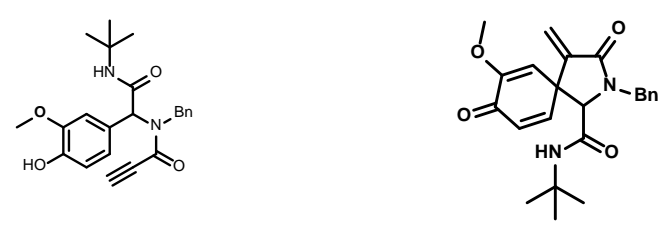

For detail analytical data: Ranjan, P.; Ojeda, G M.; Sharma, U. K.; Van der Eycken, E. V. Chem. Eur. J. 2019, 25, 2442 - 2446 
9. NMR Spectra
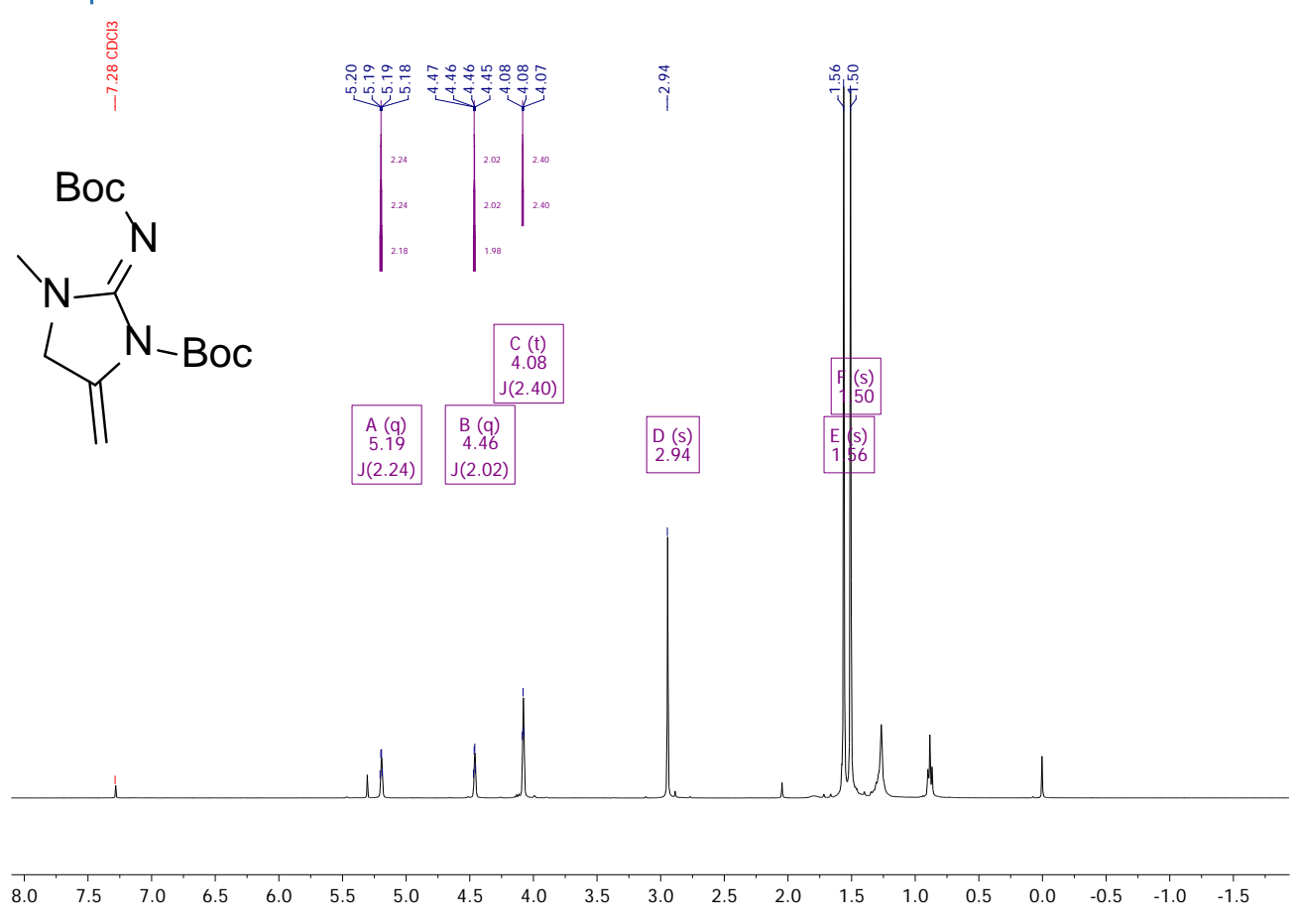

${ }^{1} \mathrm{H}-\mathrm{NMR}$ of product 2a

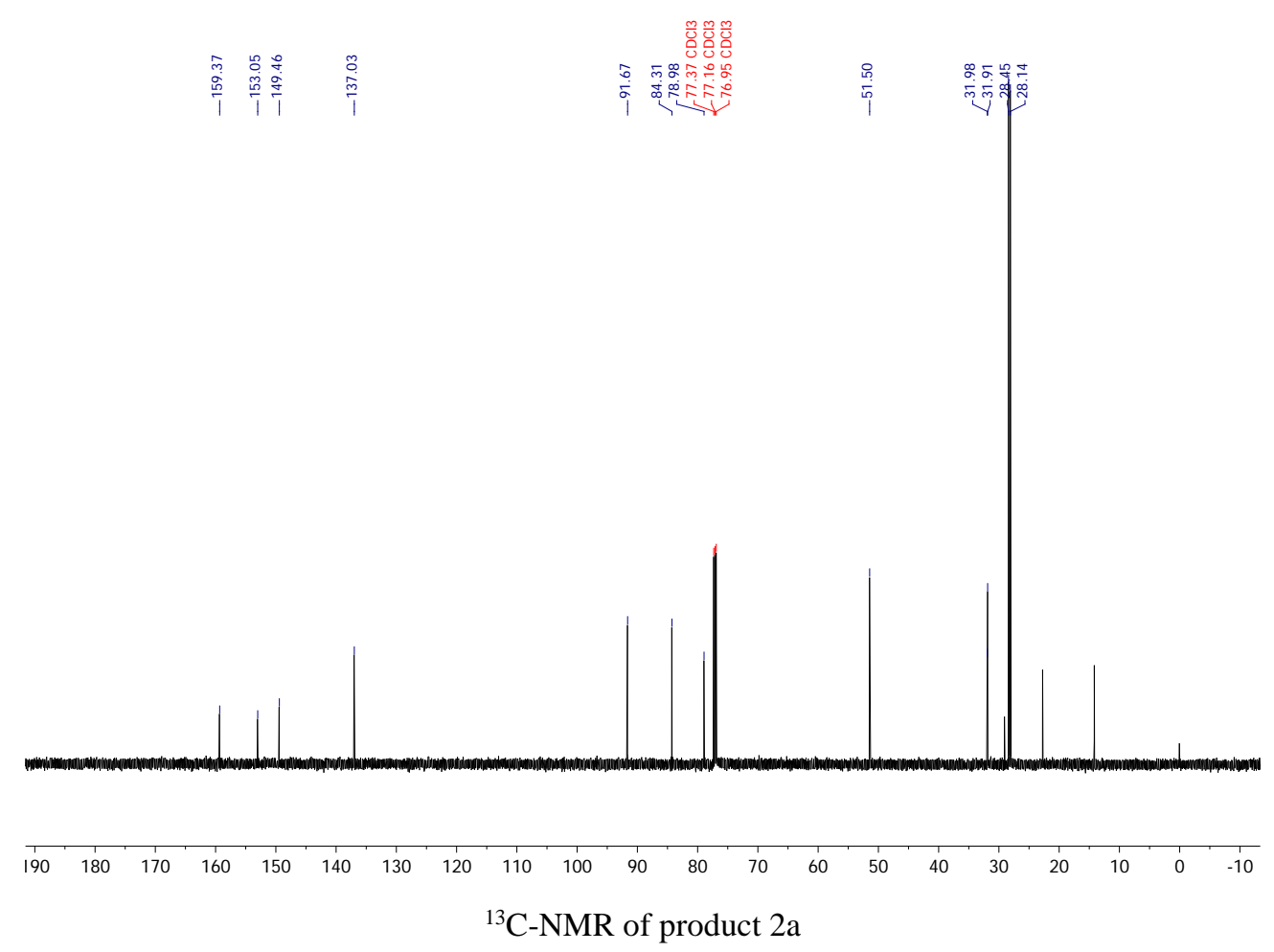




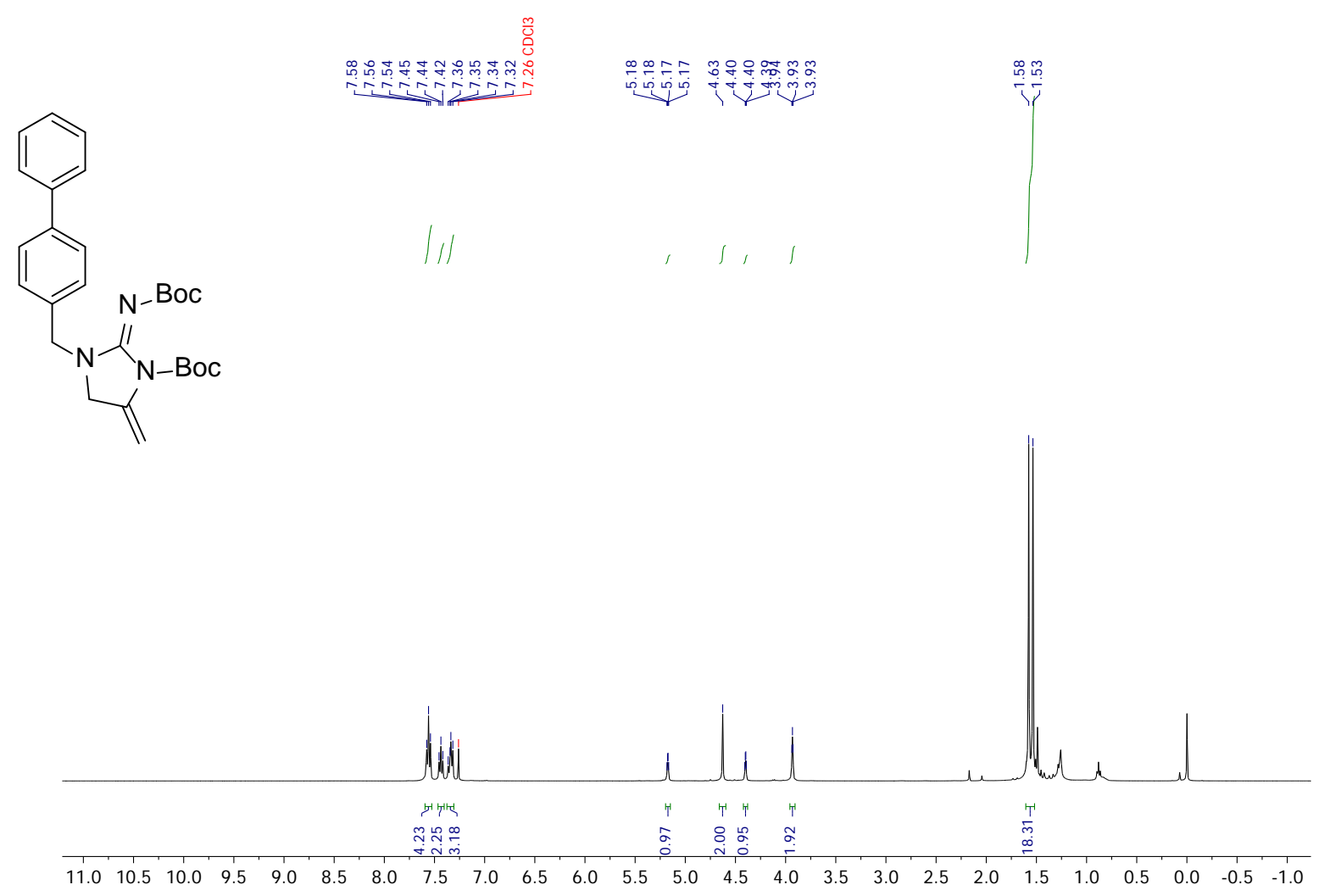

${ }^{1} \mathrm{H}-\mathrm{NMR}$ of $2 \mathrm{~b}$

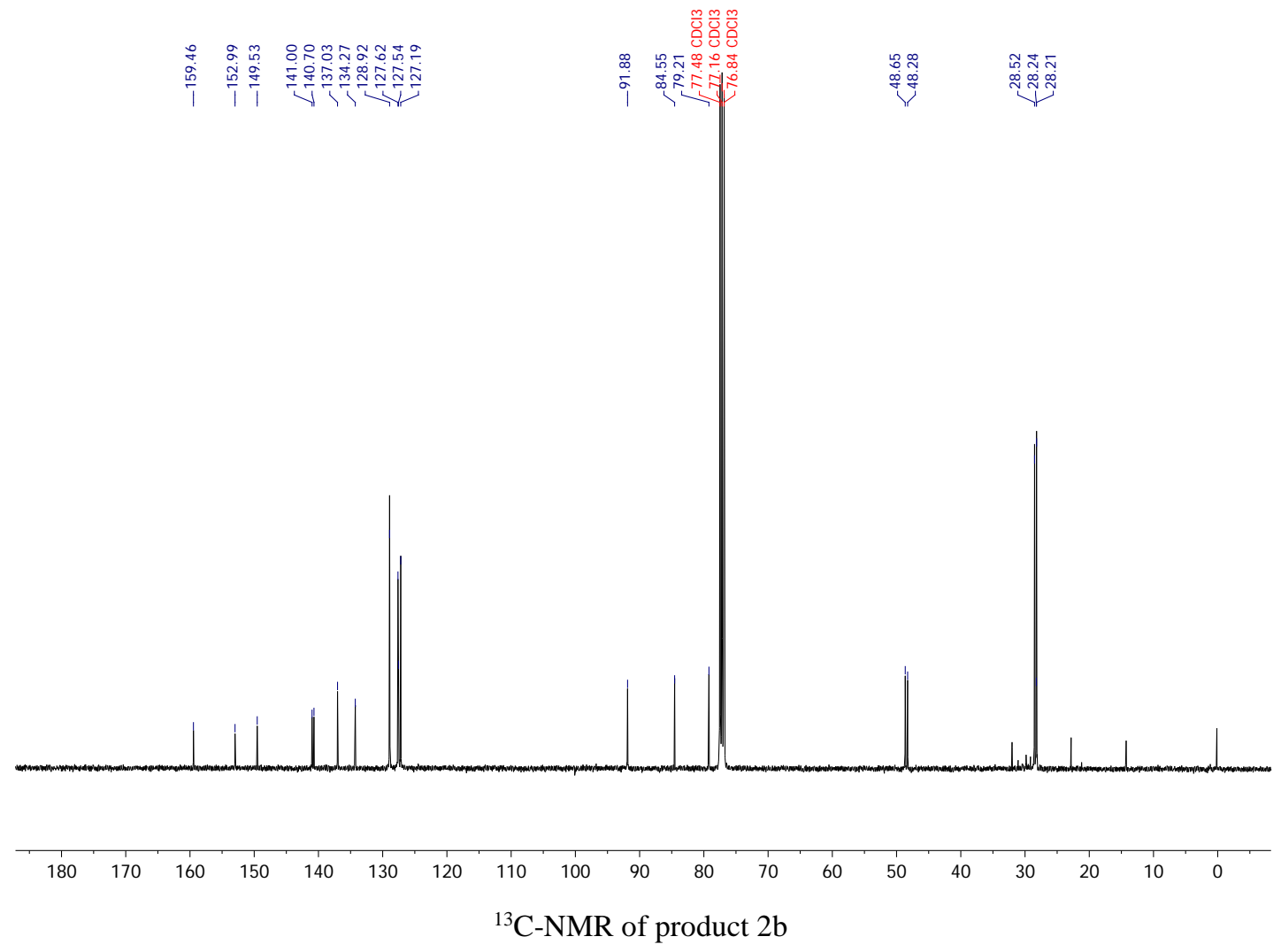


8

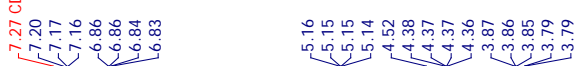

Boc

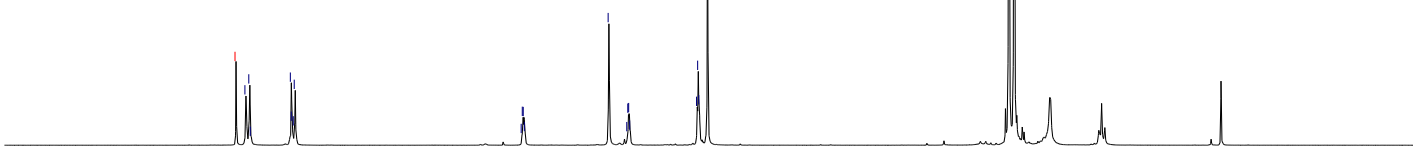

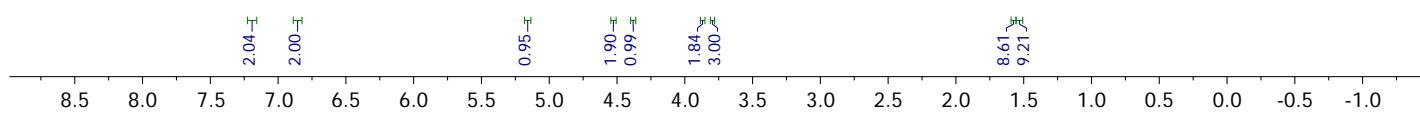

${ }^{1} \mathrm{H}-\mathrm{NMR}$ of $2 \mathrm{c}$

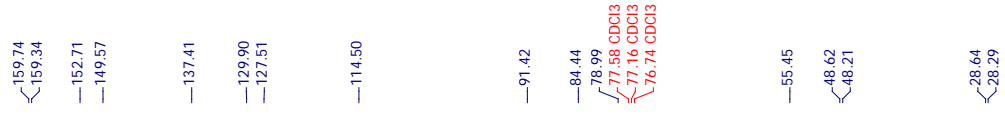

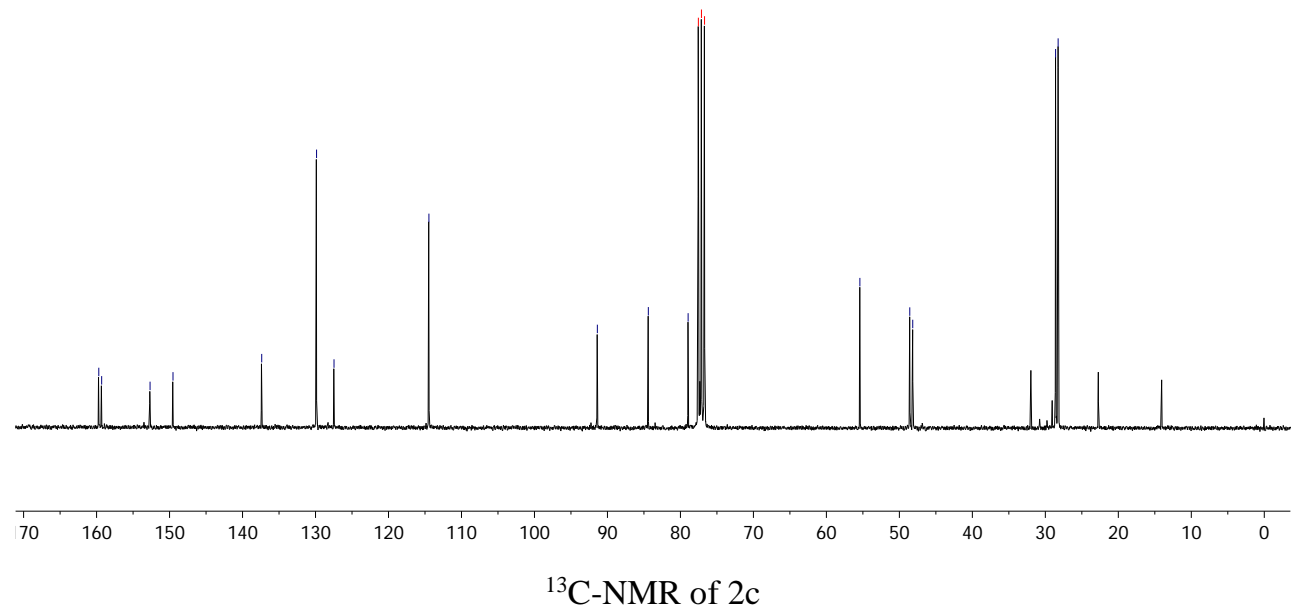




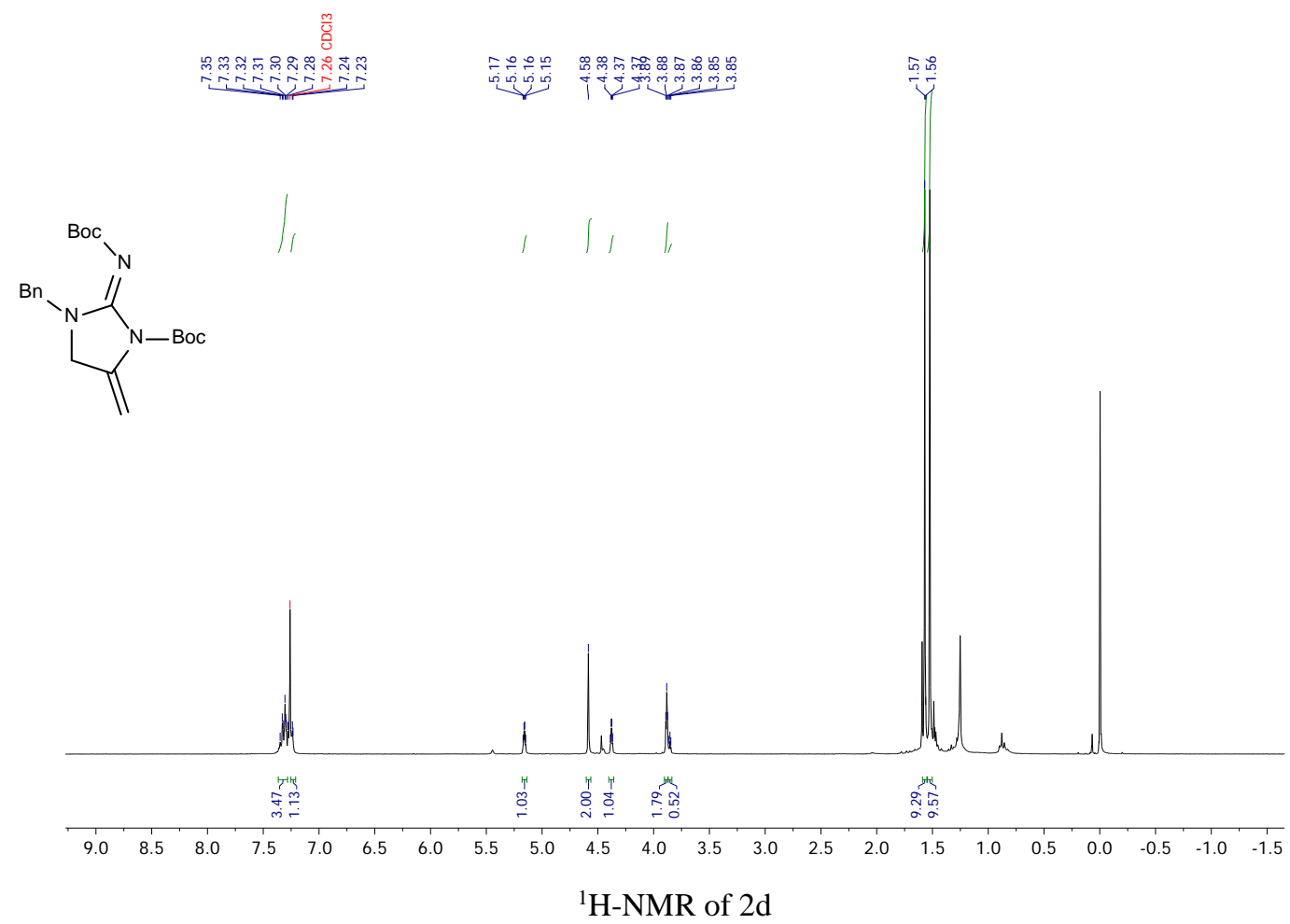

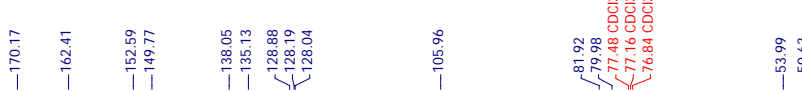

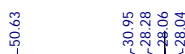

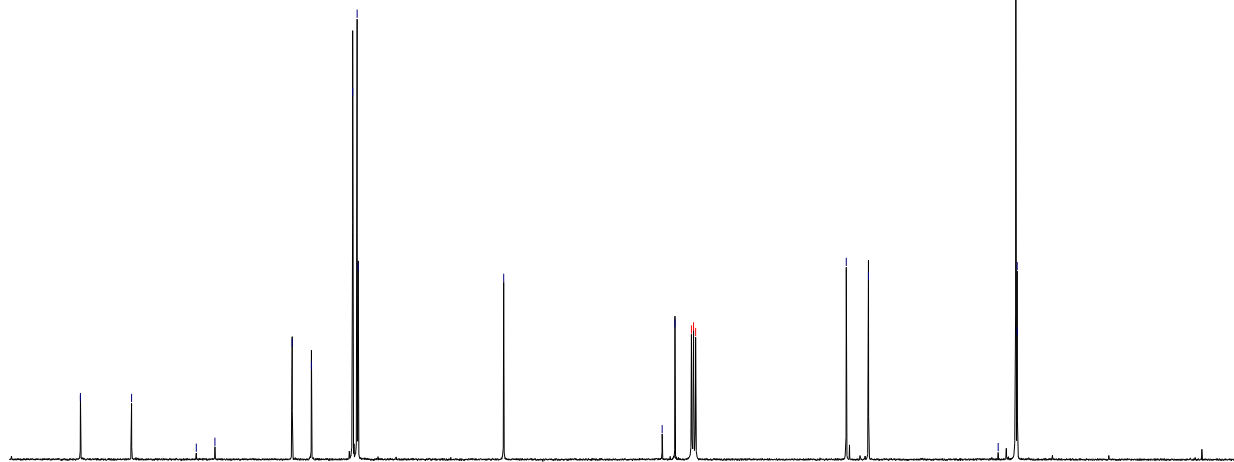

\begin{tabular}{lllllllllllllllllll}
\hline 80 & 170 & 160 & 150 & 140 & 130 & 120 & 110 & 100 & 90 & 80 & 70 & 60 & 50 & 40 & 30 & 20 & 10 & 0
\end{tabular}

${ }^{13} \mathrm{C}-\mathrm{NMR}$ of $2 \mathrm{~d}$ 


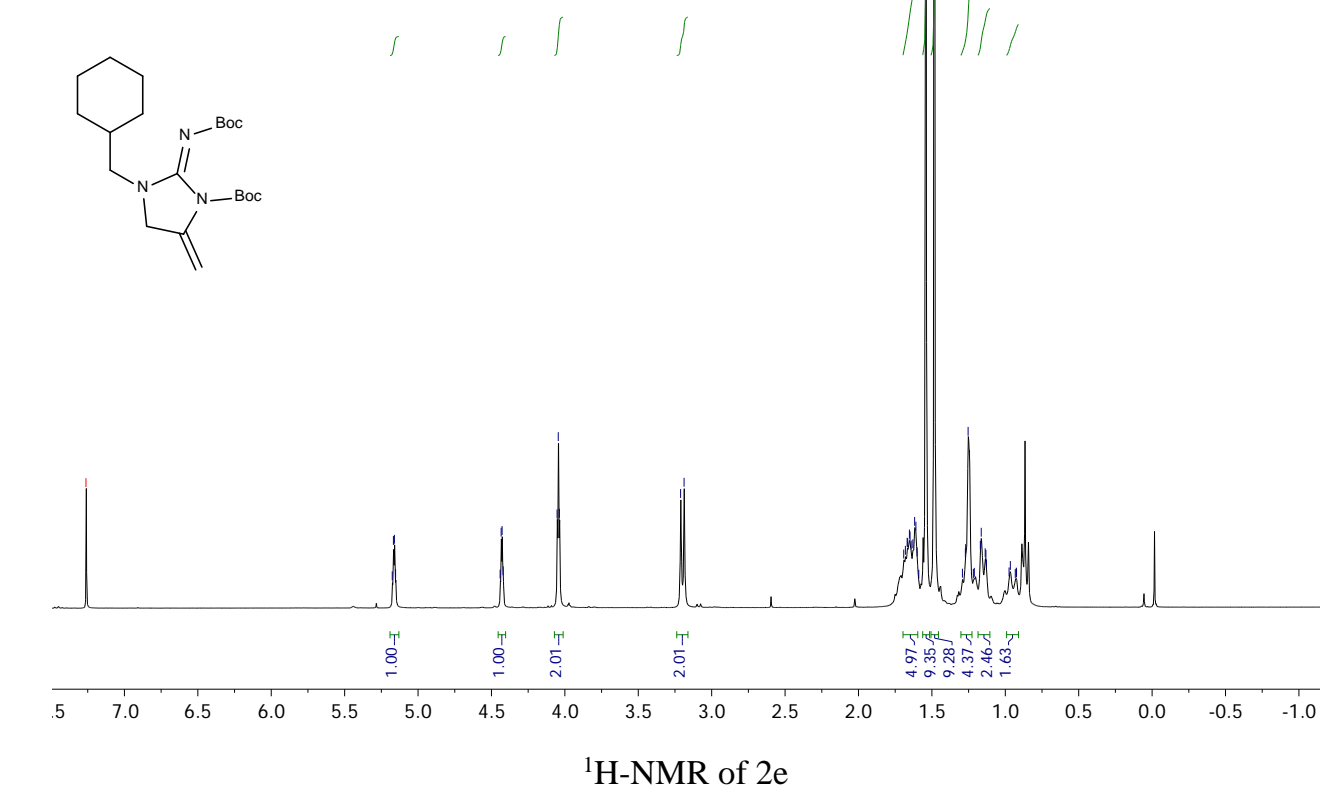

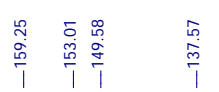

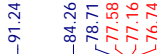

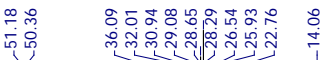
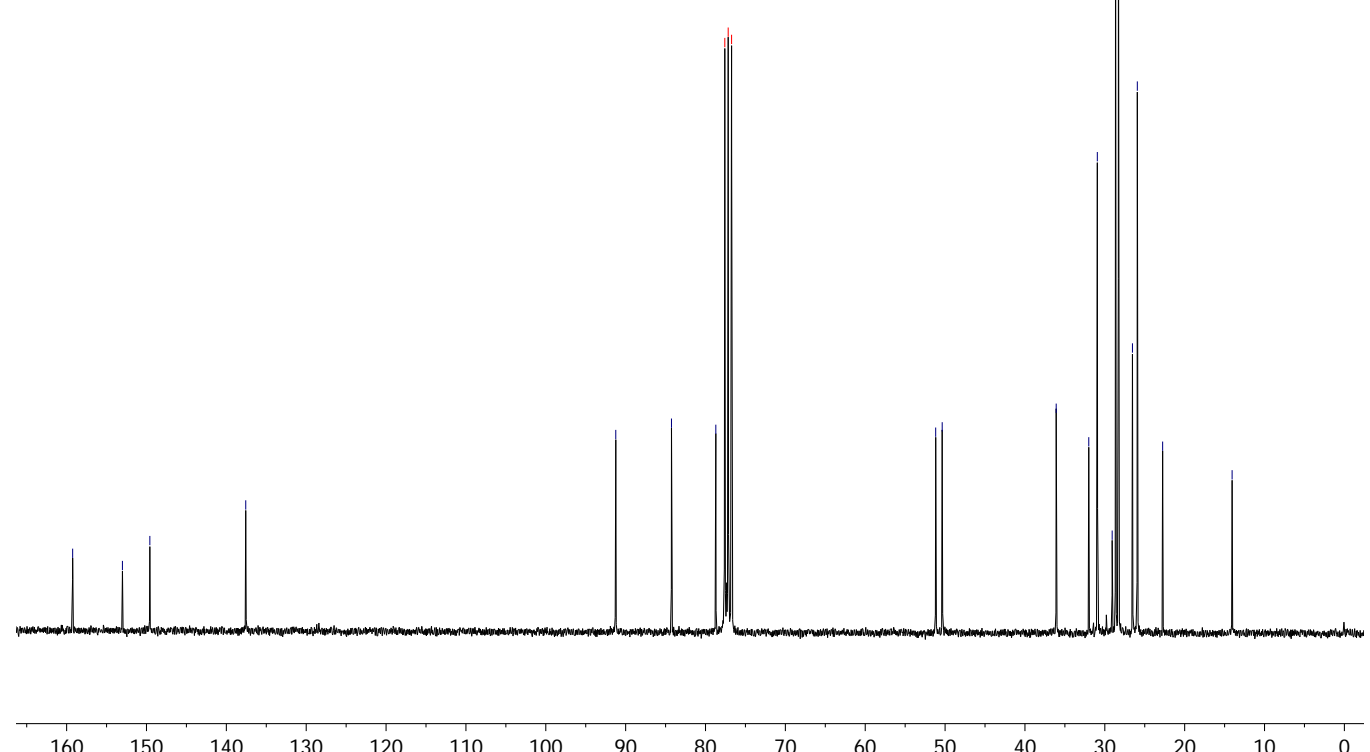

${ }^{13} \mathrm{C}-\mathrm{NMR}$ of $2 \mathrm{e}$ 

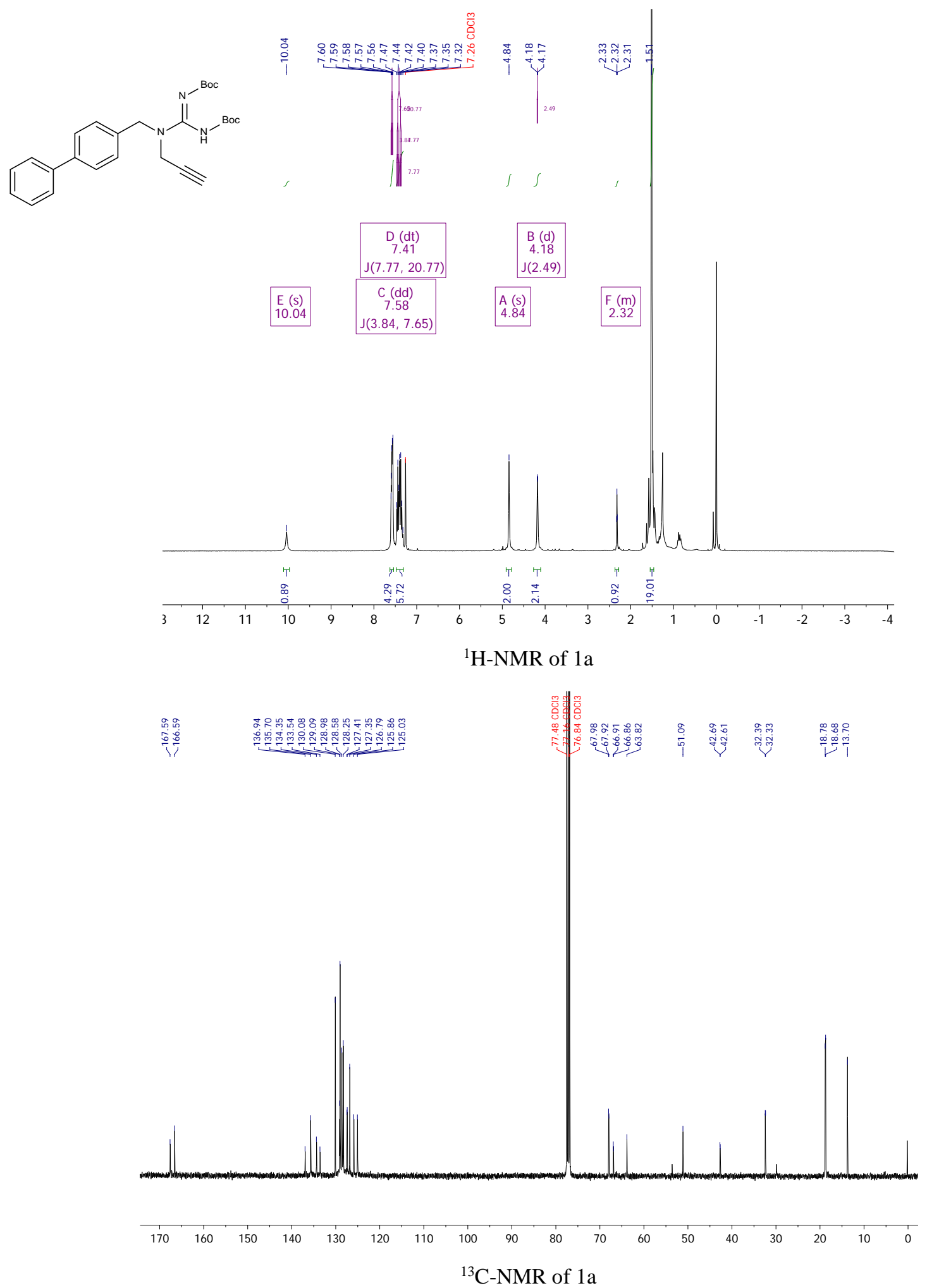


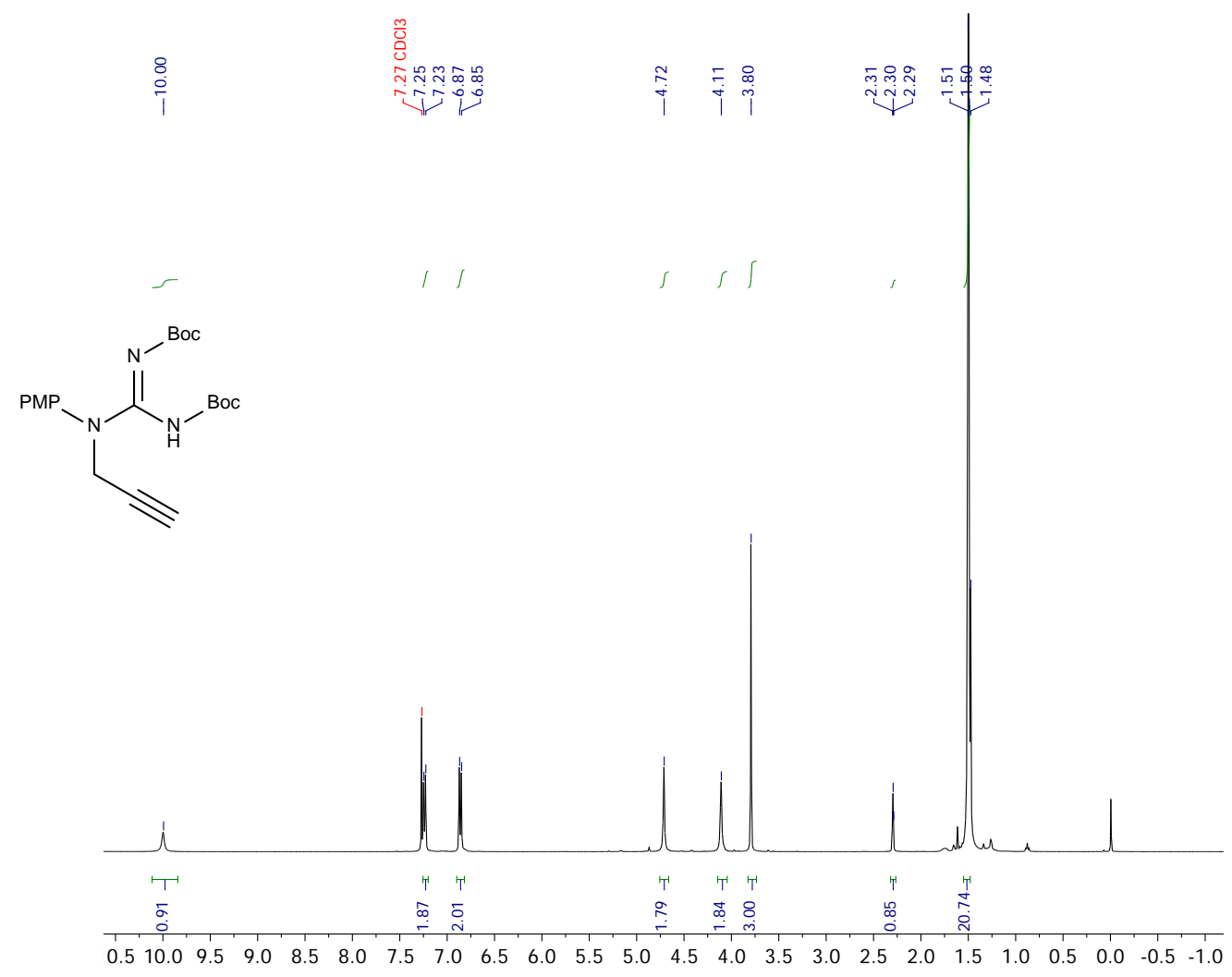

${ }^{1}$ H-NMR of $1 \mathrm{c}$

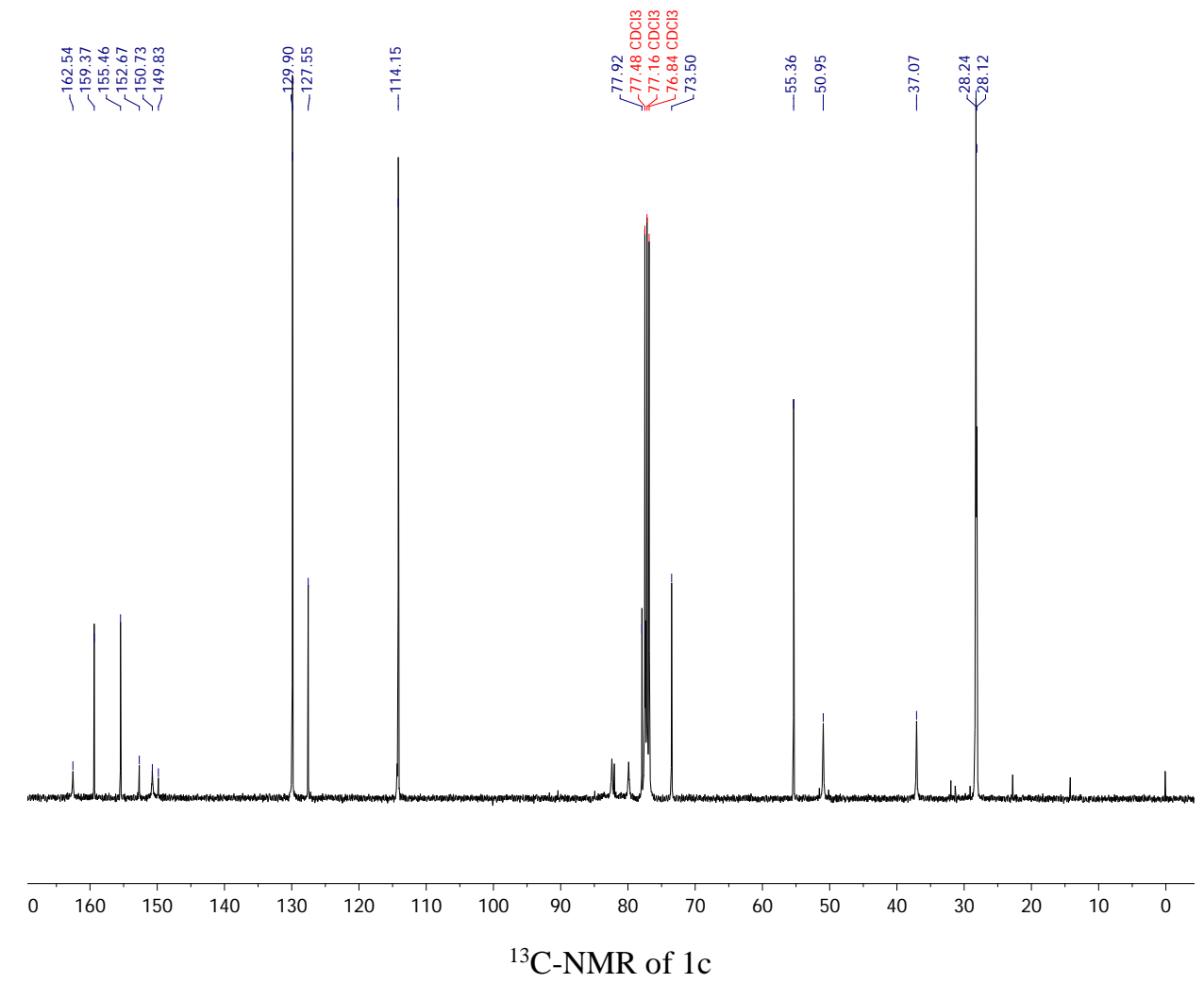




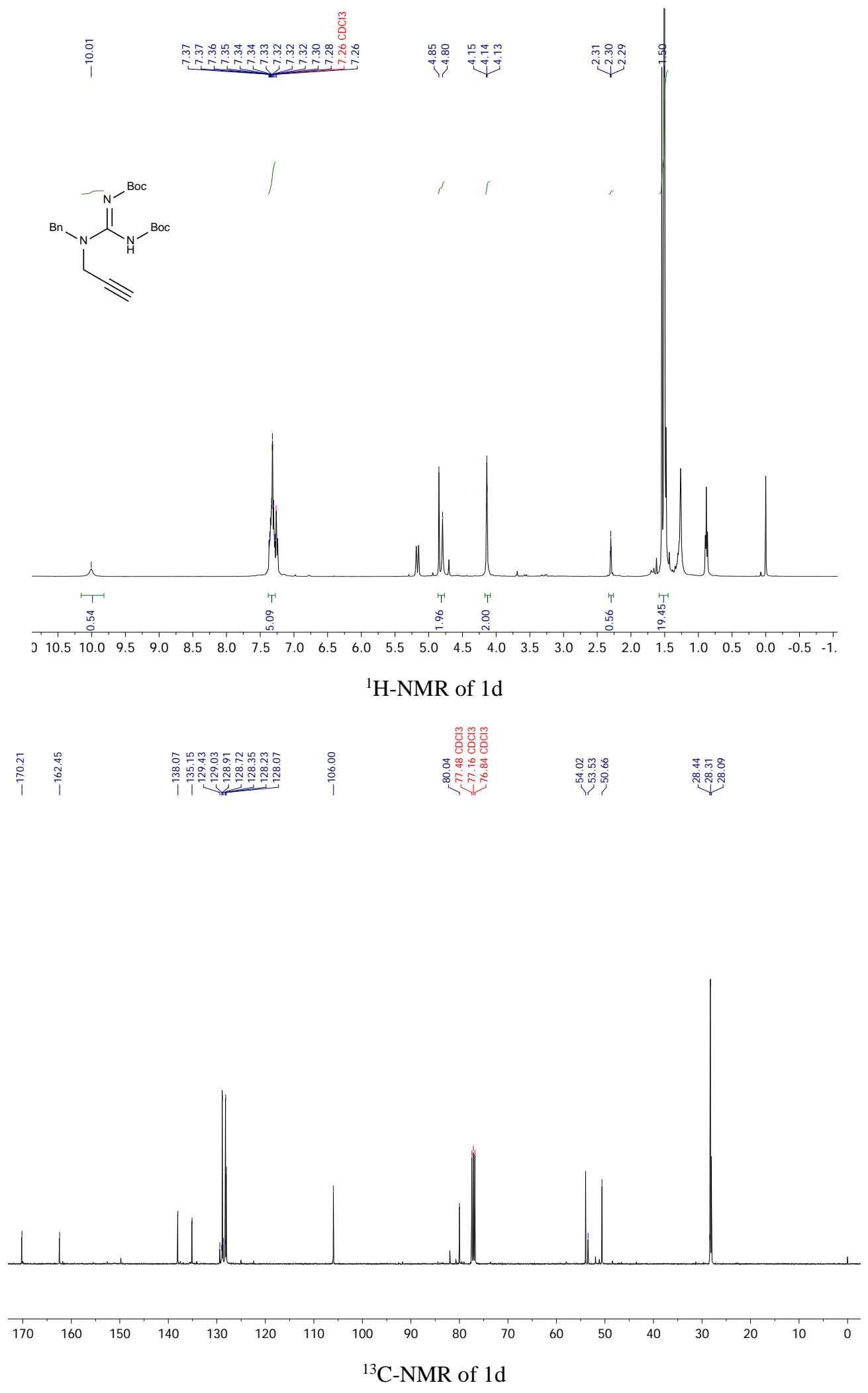




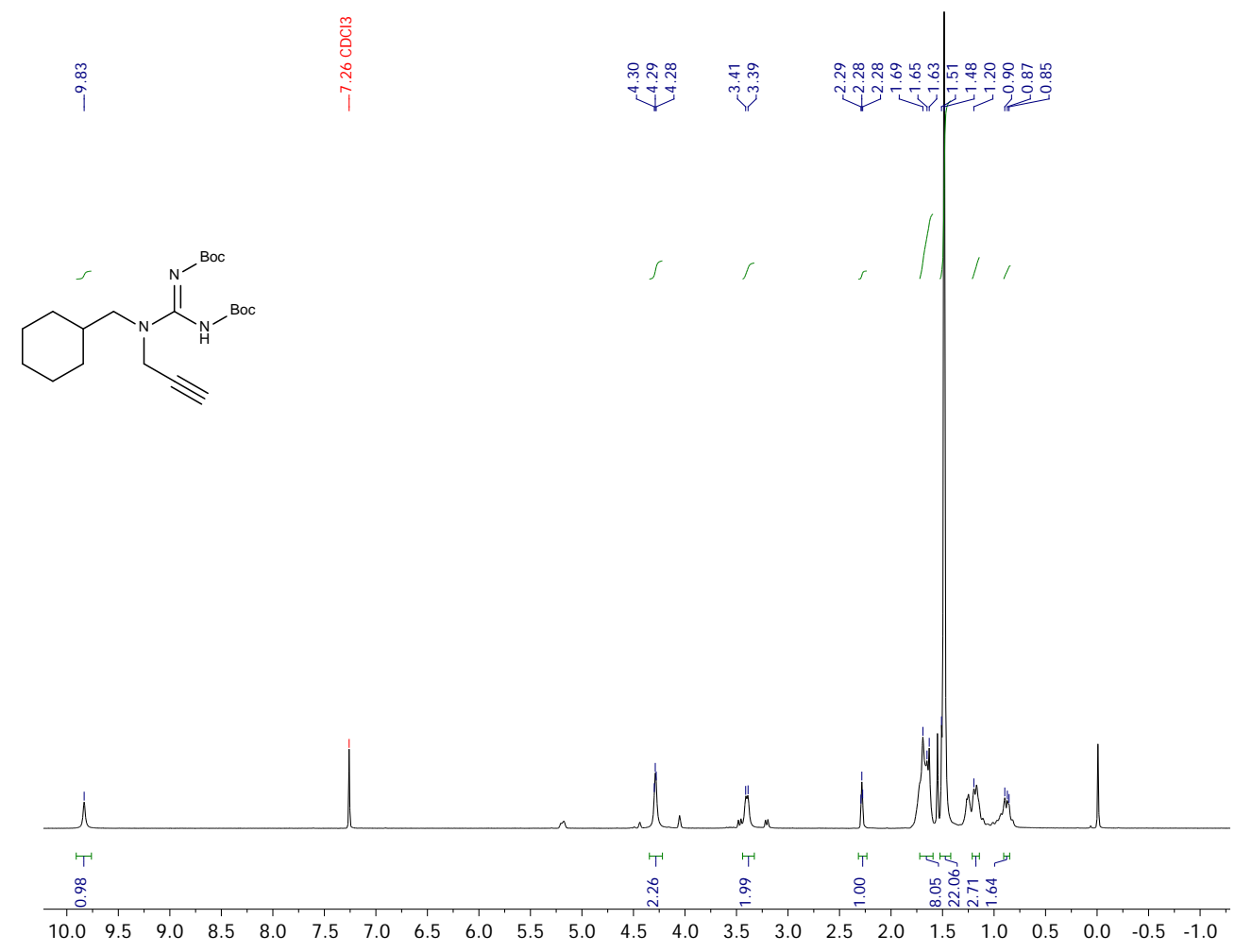

${ }^{1} \mathrm{H}-\mathrm{NMR}$ of $1 \mathrm{e}$

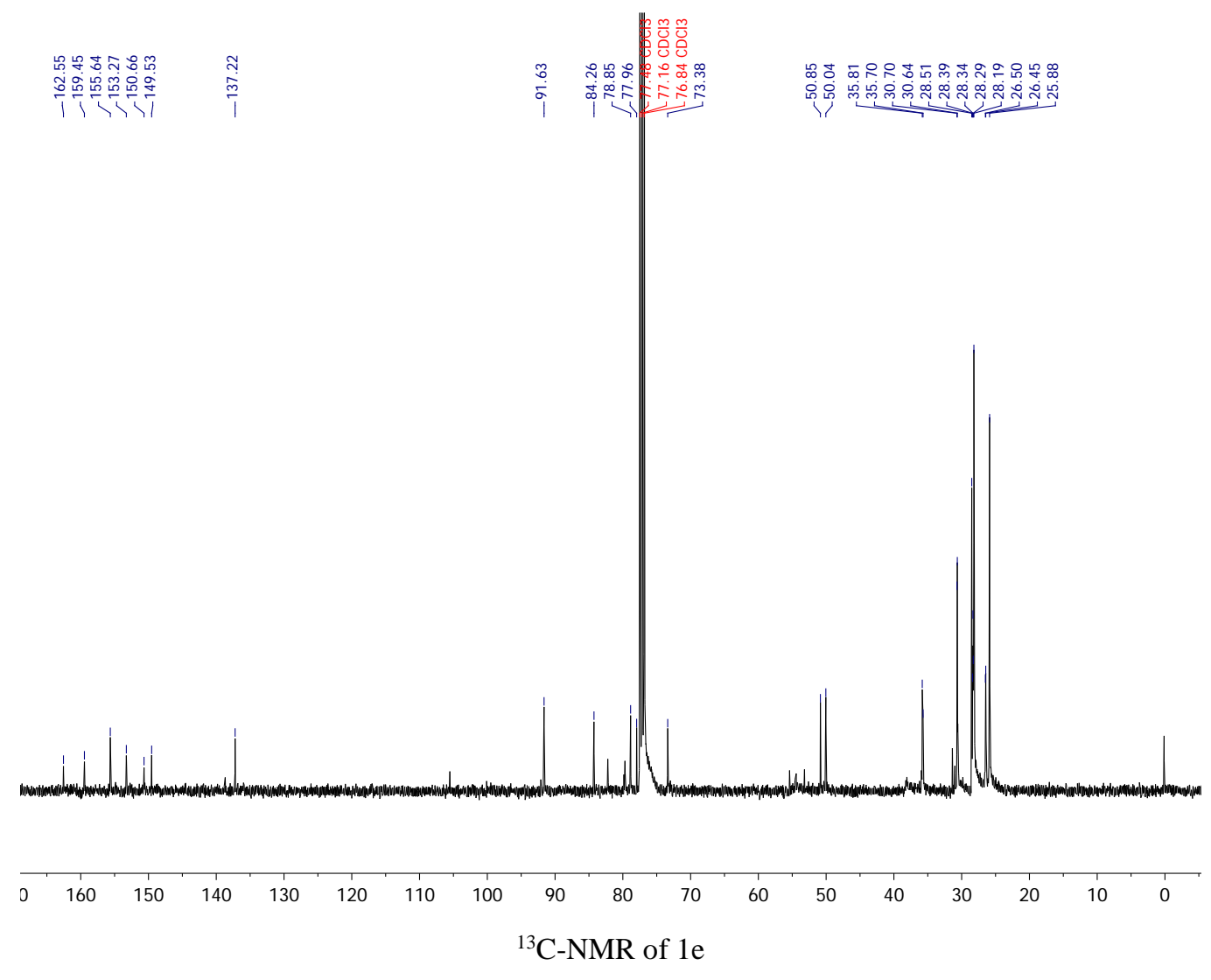




\section{REFERENCES}

(1) Liu, S.; Lu, F.; Zhu, J.-J. Highly Fluorescent Ag Nanoclusters: Microwave-Assisted Green Synthesis and Cr3+ Sensing. Chem. Commun. 2011, 47 (9), 2661-2663. https://doi.org/10.1039/c0cc04276e.

(2) Purandarea, P. S.; Leleb, M. M.; Gupta, R. Parametric Analysis of Helical Coil Heat Exchanger. Int. J. Eng. Res. Technol. 2012, 1 (8), 1-5.

(3) Manno, R.; Sebastian, V.; Mallada, R.; Santamaria, J. 110th Anniversary: Nucleation of Ag Nanoparticles in Helical Microfluidic Reactor. Comparison between Microwave and Conventional Heating. Ind. Eng. Chem. Res. 2019, 58 (28), 12702-12711. https://doi.org/10.1021/acs.iecr.9b01460.

(4) Andhare, A.; Kriplani, V. M.; Modak, J. P. Thermal Analysis of a Helical Coil Heat Exchanger. Int. J. Innov. Res. Adv. Eng. 2014, 1 (12), 137-143.

(5) Andrzejczyk, R.; Muszyński, T. Performance Analyses of Helical Coil Heat Exchangers. the Effect of External Coil Surface Modification on Heat Exchanger Effectiveness. Arch. Thermodyn. 2016, 37 (4), 137-159. https://doi.org/10.1515/aoter-2016-0032.

(6) Huang, W.; Liu, J. H.; Alayoglu, P.; Li, Y.; Witham, C. A.; Tsung, C.; Toste, F. D.; Somorjai, G. A. Highly Active Heterogeneous Palladium Nanoparticle Catalysts for Homogeneous Electrophilic Reactions in Solution and the Utilization of a Continuous Flow Reactor. J. Am. Chem. Soc. 2010, 132 (47), 16771-16773. https://doi.org/10.1021/ja108898t. 